\title{
RNA N6-methyladenosine Reader IGF2BP2 Promotes Lymphatic Metastasis and Epithelial- mesenchymal Transition of Head and Neck Squamous Carcinoma Cells via Stabilizing Slug mRNA in an M6A-dependent Manner
}

Dan Yu ( $\nabla$ yudan@stu.cqmu.edu.cn )

The First Affiliated Hospital of Chongqing Medical University

Min Pan

The First Affiliated Hospital of Chongqing Medical University

\section{Yanshi Li}

The First Affiliated Hospital of Chongqing Medical University

\section{Tao Lu}

The First Affiliated Hospital of Chongqing Medical University

\section{Zhihai Wang}

The First Affiliated Hospital of Chongqing Medical University

\section{Chuan Liu}

The First Affiliated Hospital of Chongqing Medical University

\section{Guohua $\mathrm{Hu}$}

The First Affiliated Hospital of Chongqing Medical University

\section{Research Article}

Keywords: N6-methyladenosine, IGF2BP2, Slug, EMT, lymphatic metastasis, HNSCC

Posted Date: October 7th, 2021

DOl: https://doi.org/10.21203/rs.3.rs-952536/v1

License: (c) (1) This work is licensed under a Creative Commons Attribution 4.0 International License. Read Full License

Version of Record: A version of this preprint was published at Journal of Experimental \& Clinical Cancer Research on January 3rd, 2022. See the published version at https://doi.org/10.1186/s13046-021-022121. 


\section{Abstract}

Background: Lymph node metastasis is the main cause of poor prognosis of head and neck squamous carcinoma (HNSCC) patients. N6-methyladenosine (m6A) RNA modification is an emerging epigenetic regulatory mechanism for gene expression, and as a novel m6A reader protein, IGF2BP2 has been implicated in tumor progression and metastasis. However, not much is currently known about the functional roles of IGF2BP2 in HNSCC, and whether IGF2BP2 regulates lymphatic metastasis through m6A modification in HNSCC remains to be determined.

Methods: The expression and overall survival (OS) probability of m6A-related regulators in HNSCC were analyzed with The Cancer Genome Atlas (TCGA) dataset and GEPIA website tool, respectively. The expression levels of IGF2BP2 were measured in HNSCC tissues and normal adjacent tissues. To study the effects of IGF2BP2 on HNSCC cell metastasis in vitro and in vivo, gain- and loss- of function methods were employed. RIP, MeRIP, and luciferase reporter and mRNA stability assays were performed to explore the epigenetic mechanism of IGF2BP2 in HNSCC.

Results: We investigated 20 m6A-related regulators in HNSCC and discovered that only the overexpression of IGF2BP2 was associated with a poor OS probability and an independent prognostic factor for HNSCC patients. Additionally, we demonstrated that IGF2BP2 was overexpressed in HNSCC tissues, and significantly correlated to lymphatic metastasis and poor prognosis. Functional studies have shown that IGF2BP2 promotes both HNSCC cell migration as well as invasion via the epithelialmesenchymal transition (EMT) process in vitro, and IGF2BP2 knockdown significantly inhibited lymphatic metastasis and lymphangiogenesis in vivo. Mechanistic investigations revealed that Slug, a key EMT-related transcriptional factor, is the direct target of IGF2BP2, and essential for IGF2BP2-regulated EMT and metastasis in HNSCC. Furthermore, we demonstrated that IGF2BP2 recognizes and binds the m6A site in the coding sequence (CDS) region of Slug and promotes its mRNA stability.

Conclusions: Collectively, our study uncovers the oncogenic role and potential mechanism of IGF2BP2, which serves as a m6A reader, in controlling lymphatic metastasis and EMT in HNSCC, suggesting that IGF2BP2 may act as a therapeutic target and prognostic biomarker for HNSCC patients with metastasis.

\section{Background}

Head and neck squamous cell carcinoma (HNSCC), which can develop in the larynx, pharynx, and oral cavity, ranks as the sixth leading malignancy worldwide, resulting in approximately 350,000 deaths each year $[1,2]$. The majority of newly diagnosed patients with HNSCC present at the locally advanced stages and the majority of these patients have regional lymph node (LN) metastasis at the onset [2,3]. Despite recent advances in therapeutic approaches, such as multidisciplinary treatment and targeted immunotherapy [4-7], the 5-year overall survival (OS) rate of HNSCC patients is still only $\mathbb{8 0} \%$, mainly owing to the high rate of lymphatic metastasis and postoperative recurrence [2-4]. Therefore, clarifying 
the molecular mechanisms that underlie HNSCC initiation and metastasis is of great importance for the improvement in patient prognosis and development of targeted therapeutic strategies.

An RNA binding protein (RBP) known as the insulin-like growth factor 2 mRNA-binding protein 2 (IGF2BP2) is related to the regulation of various cellular and biological processes [8, 9]. Abnormal expression of IGF2BP2 is commonly linked to multiple diseases, including cancers [10,11]. Accumulating evidence has revealed the oncogenic role of IGF2BP2 in tumorigenesis and metastasis [12-15]. Experiments with IGF2BP2-deficient mice has indicated that IGF2BP2 is a tumor-promoting factor that facilitates the progression of cancer as well as metastasis [16]. Meanwhile, IGF2BP2 overexpression induces genome instability and promotes cancer cell proliferation and migration in vitro $[13,17,18]$. It has been demonstrated recently that proteins of the IGF2BP family (IGF2BPs, including IGF2BP2) are a distinct type of $\mathrm{N}^{6}$-methyladenosine $(\mathrm{m} 6 \mathrm{~A})$ readers, which are capable of regulating and recognizing the m6A modification of target mRNAs and crucial for their oncogenic functions [19].

Among the hundreds of internal RNA modifications, m6A is considered to be the most prevalent type in eukaryotic mRNA [20-22]. The m6A modification is reversible, and is catalysed by methyltransferases ("writers") and removed by demethylases ("erasers") [20,23]. In this dynamic process, the fate and biological function of m6A-modified RNAs mainly relies on the m6A binding proteins ("readers") [23, 24]. "Readers" are those that can recognize and bind directly to m6A modification sites, and exert more specific regulatory functions, including alternative splicing, stability of mRNA, RNA processing, and translation [25, 26]. IGF2BPs and the YT521-B homology (YTH) domain family (YTHDF) are the two crucial RBP families of m6A "readers" [26, 27]. Different from YTHDF that may accelerate mRNA decay [27], IGF2BPs, as newly identified m6A readers, can target numerous mRNAs and maintain their stability to promote cancer progression and metastasis in a m6A-dependent manner [28, 29]. Several recent studies have reported that m6A modification is implicated in lymphatic metastasis in cancers [30-32]. However, the functional roles of IGF2BP2 in HNSCC, and more specifically whether IGF2BP2 regulates lymphatic metastasis in HNSCC by modifying m6A is currently unclear.

The primary cause of over $90 \%$ of mortality related to cancer is tumor metastasis [33,34], and epithelialmesenchymal transition (EMT) has been proven to be the initial crucial factor in tumor metastasis [35]. The process of EMT is characterized by the loss of cell adhesion and epithelial markers, like E-cadherin, which is a fundamental event for EMT; and results in the gain of enhanced invasive capacities and mesenchymal markers, including N-cadherin and vimentin [36]. EMT is mediated by a variety of EMT transcriptional factors (EMT-TFs), including Snail, Slug, ZEB1, and Twist, which can inhibit the expression of E-cadherin to promote the motility and invasiveness of cancer cells [37, 38]. Slug, encoded by the SNAI2 gene, is a key transcriptional factor for EMT that orchestrates a variety of biological processes critical to tumorigenesis and metastasis $[39,40]$. Several studies have demonstrated that Slug can be regulated by RNA binding proteins at the post-transcriptional level, promoting the mesenchymal cell properties and metastasis of tumor cells [41-43]. However, whether IGF2BP2 regulates Slug expression via m6A modification and affects the process of EMT in cancer cells still needs to be elucidated. 
In this study, we report a direct correlation between increased expression of IGF2BP2 with lymphatic metastasis and decreased survival of HNSCC patients. Our results show that knockdown of IGF2BP2 resulted in a significant reduction in lymphatic metastasis and lymphangiogenesis in a popliteal LN metastasis model. Mechanistically, we identified that Slug is the direct target mRNA of IGF2BP2 and is critical to the EMT process and metastasis in HNSCC. More importantly, we demonstrated that IGF2BP2 recognizes and binds the m6A site in the coding sequence (CDS) of Slug and stabilizes its mRNA.

\section{Methods}

\section{TCGA data mining and analysis tools}

RNA-sequencing data (level 3) in raw form and related clinical data regarding HNSCC were derived online from The Cancer Genome Atlas (TCGA) dataset (https://portal.gdc.cancer.gov/), which contained 502 HNSCC cases and 44 normal cases. Analysis and visualization of the genes' expression was conducted with R software (version 4.0.3) packages 'ggplot2' and 'pheatmap'. The GEPIA website tool (http://gepia2.cancer-pku.cn/\#index) was used to conduct the OS analysis. Analysis and visualization of the Kaplan-Meier survival analysis was performed with the R software packages 'survival' and 'survminer'. Multivariate cox regression analysis was conducted in order to determine the fitting terms, which were used for building the nomogram. The 'forestplot' package, found in $\mathrm{R}$, was used to identify each variable's $P$ value, hazard ratio $(\mathrm{HR})$, and $95 \%$ confidence interval $(\mathrm{Cl})$. To predict the 1-, 3-, and 5-year overall recurrence, we developed a nomogram in conformity with the results of the multivariate Cox proportional hazards analysis. The nomogram is a visual representation of factors, and can be used for calculating the risk of recurrence for a distinct patient through summing up the points correlated to each risk factor in the 'rms' package of R. The two-gene correlation map was constructed with the 'ggstatsplot' package in $\mathrm{R}$ software based on Spearman's correlation analysis.

\section{HNSCC patients and clinical samples}

For this study, 20 paired fresh HNSCC tissues and normal adjacent tissues (NATs), and 20 fresh HNSCC tissues with or without lymphatic metastasis (consisting of 10 cases with lymphatic metastasis and 10 cases without lymphatic metastasis) were retrieved from HNSCC patients who received surgery at the Department of Otorhinolaryngology of the First Affiliated Hospital of Chongqing Medical University (Chongqing, China). Fresh tissues were washed with saline after resection, and liquid nitrogen was used to freeze them immediately after which they were kept at $-80^{\circ} \mathrm{C}$ for total protein and RNA extraction. Furthermore, in total 78 HNSCC tissue specimens embedded in paraffin were collected from the Department of Pathology of the First Affiliated Hospital of Chongqing Medical University. The 78 HNSCC patients underwent surgery between January 2012 and December 2019 in the Otolaryngology department of the First Affiliated Hospital of Chongqing Medical University. The clinical features of the patients are detailed in Table 1. Informed consent was acquired from all patients before surgery. The study was 
authorized by the Biomedical Ethics Committee of the First Affiliated Hospital of Chongqing Medical University.

\section{Cell lines and cell culture}

FaDu cells were obtained from the Chinese Academy of sciences (Shanghai, China). SCC15 cells were the kind gift of Prof. Kai Yang (Chongqing Medical University, China). FaDu and SCC15 cells were both kept in Dulbecco's modified Eagle's medium (DMEM, Gibico, USA), to which $10 \%$ fetal bovine serum (FBS, PANBiotech, Germany) and $1 \%$ penicillin-streptomycin was added, in an atmosphere of $37^{\circ} \mathrm{C}$ and $5 \% \mathrm{CO}_{2}$.

\section{Immunohistochemistry (IHC) staining and scoring analyses}

IHC was performed with a detection kit (SP-9000, Beijing, Zhongshan Jinqiao) in accordance with the manufacturer's instructions. In short, fresh xylene was used to deparaffinize the paraffin sections, which were then hydrated in gradient alcohol. The citric acid buffer was used for antigen retrieval at $90^{\circ} \mathrm{C}-100^{\circ} \mathrm{C}$ for 30 minutes to which an appropriate amount of endogenous peroxidase blocker was added. The standard goat serum working solution was sealed at room temperature for 15 minutes, and then transferred to a refrigerator of $4^{\circ} \mathrm{C}$ and incubated with IGF2BP2 and LYVE-1 primary antibodies overnight. On the second day, horseradish enzyme-labeled streptavidin was dripped into the working solution, then incubated for 15 min at room temperature, and rinsed with PBS 3 times for 3 minutes each time. Diaminobenzidine (DAB) was used for color development, followed by hematoxylin staining for 30 seconds, and rinsing with tap water for 5 minutes. Finally, it was dehydrated, transparent and mounted with neutral gum. The IHC stained sections were reviewed and scored independently by two superior pathologists. A final score was then calculated by multiplying the signaling intensity score and the staining distribution score. The signal intensity scores were categorized as follows: 0 (no signal), 1 (weak), 2 (moderate), and 3 (strong). The staining distribution scores were determined according to the percentage of positive cells: $0(0 \%), 1(1-10 \%), 2(10-50 \%)$, and $3(51-100 \%)$.

\section{Extraction of RNA and quantitative real-time PCR (qRT-PCR)}

An E.Z.N.A. ${ }^{\circledR}$ Total RNA Kit I (Omega Bio-tek, USA) was utilized for isolation of the total RNA from HNSCC specimens and cells according to instructions of the manufacturer. Subsequently, an Agilent 2100 Bioanalyzer (Agilent, Santa Clara, CA, USA) was used to determine the total RNA's quality through absorbance readings at $260 \mathrm{~nm}$. Reverse transcription of total RNA into complementary DNA (CDNA) was done with a gDNA Eraser (TaKaRa, Japan) using a PrimerScriptTM RT Reagent Kit. Next, for the amplification process an ABI 7500 Real-Time PCR System (Applied Biosystems, Foster City, CA, USA) and a SYBR Premix Ex TagTM Kit (TaKaRa, Japan) were utilized in accordance with instructions of the manufacturer. The conditions in which the PCR amplification was conducted was as follows: 40 cycles in total at $95^{\circ} \mathrm{C}$ for the duration of $30 \mathrm{~s}$, then $95^{\circ} \mathrm{C}$ for $5 \mathrm{~s}$, and lastly $60^{\circ} \mathrm{C}$ for 1 min. Glyceraldehyde 3- 
phosphate dehydrogenase (GAPDH) functioned as internal control. The $2^{-\Delta \Delta C t}$ method was applied for analysis of the results, which were presented as relative expression. All experiments were performed three times to independently verify the findings. The primers for PCR analysis are listed in Additional file 1: Table S1.

\section{Protein extraction and western blotting analysis}

Total proteins from HNSCC specimens and cells were derived with a protein extraction kit (KGP250, KeyGen, Jiangsu, China). A bicinchoninic acid (BCA) protein assay kit (P0010S, Beyotime, Shanghai, China) was utilized for the detection of the concentration of protein. Next, protein extracts were diluted in $5 \times$ loading buffer and boiled for $10 \mathrm{~min}$. The boiled proteins were subjected to $10 \%$ SDS-PAGE, placed onto polyvinylidene fluoride (PVDF) membranes, and blocked with $5 \%$ nonfat dry milk for the duration of $2 \mathrm{~h}$. Subsequently, we incubated the membranes overnight at $4{ }^{\circ} \mathrm{C}$ with primary antibodies, of which the details are shown in Additional file 2: Table S1. The next day, horseradish peroxidase (HRP)-conjugated secondary antibodies were used for incubating the membranes for $1 \mathrm{~h}$ in total at room temperature. Finally, an enhanced chemiluminescence (ECL) kit (12043-D10, Advansta, USA) was utilized to visualize the protein blots, of which the images were taken with the ChemiDoc Touch Imaging System (Bio-Rad, USA) and analyzed by ImageJ software (version v1.8.0).

\section{siRNA and cell transfection}

To reduce the off-target effect of the siRNAs, three independent siRNAs targeting IGF2BP2 and a negative control siRNA were constructed and generated by GenePharma (Shanghai, China). In brief, HNSCC cells were seeded, at a concentration of $2 \times 10^{5}$ cells/well, in six-well plates with culture medium, and incubated till 60\%-70\% confluence. Then, Lipofectamine iMAX Reagent (Invitrogen, USA) was utilized for transfection of the cells with siRNAs at a ratio of 1:3 and incubated for $24 \mathrm{~h}$ in serum-free medium. $24 \mathrm{~h}$ later, we discarded the transfection medium, and fresh complete culture medium without siRNA and Lipofectamine iMAX was added. At the timeframe of $48 \mathrm{~h}$ or $72 \mathrm{~h}$ following transfection, we collected the cells for protein or RNA extraction to conduct RT-qPCR and western blotting to validate the transfection efficiency. The IGF2BP2 target and negative control sequences are listed in Additional file 1: Table S1.

\section{Lentivirus vector and cell infection}

For knockdown, short hairpin RNA (shRNA) of human IGF2BP2 was cloned into a hU6-MCS-Ubiquitinfirefly_Luciferase-IRES-puromycin lentiviral vector (GV344, Genechem, Shanghai, China). To achieve overexpression, human full-length cDNA of IGF2BP2 was cloned into a Ubi-MCS-3FLAG-CBh-gcGFP-IRESpuromycin lentiviral vector (GV492, Genechem, Shanghai, China). The target sequences of IGF2BP2 have been detailed in Additional file 1: Table S1. When HNSCC cells had reached the logarithmic growth phase, the cells were seeded in a six-well plate at a concentration of $5 \times 10^{4}$ cells/well and incubated until roughly 
$30 \%$ confluence was reached. Then, the cells were infected with shRNA or overexpression lentiviral vectors with HiTransG A infection-enhancing solution according to the multiplicity of infection (MOI, $\mathrm{MOI}=10$ ). After incubating for $16 \mathrm{~h}$ of at $37^{\circ} \mathrm{C}$, the viral medium was discarded and substituted by fresh culture. Then, the cells were screened in culture medium containing puromycin $(2 \mu \mathrm{g} / \mathrm{ml})$ for one week and stably silenced- and overexpressed-IGF2BP2 HNSCC cells were generated.

\section{Wound-healing and Transwell assays}

Wound-healing and transwell assays were carried out in vitro for the detection of cell migration and invasion. The detailed procedures of these assays have been described in our previous study [44].

\section{Popliteal lymphatic metastasis model in vivo.}

Male BALB/cA nude mice aged 4- 6 weeks and with a weight between 18 and $22 \mathrm{~g}$ were obtained from Huafukang Biotechnology Co., (Beijing, China), and accommodated in specific pathogen free (SPF) barrier facilities. To construct the metastasis model, $5 \times 10^{6} \mathrm{FaDu}$ cells were transfected with sh-IGF2BP2luc and sh-NC-luc, suspended in $60 \mu \mathrm{lPBS}$, and then injected into the footpads of the mice. Six weeks after injection, mice were subjected to bioluminescence imaging to evaluate lymphatic metastasis. For bioluminescence imaging, mice were anesthetized by inhaling $2 \%$ isoflurane for approximately 5 min, injected intraperitoneally with D-Luciferin potassium salt $(200 \mu \mathrm{l}, 150 \mu \mathrm{g} / \mathrm{ml}, \mathrm{ST} 196$, Beyotime, Shanghai, China), and imaged with a bioluminescence system (NightOwl II LB983, Berthold Technologies, Germany). All the primary tumors and popliteal LNs were harvested and embedded in paraffin for IHC analysis. The $L N$ volumes were calculated based on this formula: $L N$ volume $\left(\mathrm{mm}^{3}\right)=$ length $\times$ width $^{2} \times$ 0.5. All the experiments were authorized by the Laboratory Animal Use Management Committee of the Experimental Animal Center of Chongqing Medical University.

\section{Immunofluorescence}

FaDu and SCC15 cells seeded on sterile coverslips were fixated with $4 \%$ paraformaldehyde for the duration of $20 \mathrm{~min}$, then permeabilized with $01 \%$ Triton X-100 for $15 \mathrm{~min}$, and lastly blocked with goat serum at room temperature for a total of $30 \mathrm{~min}$. Subsequently, primary antibodies were utilized for incubation of the cells overnight at $4^{\circ} \mathrm{C}$, of which the details are shown in Additional file 2: Table S1. The following day, fluorescence-labeled secondary antibodies were utilized incubation of the cells for $1 \mathrm{~h}$ at room temperature. DAPI (C1006, Beyotime, Shanghai, China) was used to stain nuclear DNA and the images were captured with a confocal system (Nikon).

\section{RNA binding protein immunoprecipitation (RIP)}


HNSCC cells at $90 \%$ confluence, cultured in $10-\mathrm{cm}$ plates (approximately $1.2 \times 10^{7}$ cells each plate), were accumulated and then lysed with IP lysis buffer (P0013J, Beyotime, Shanghai, China) supplemented with protease inhibitor $(100 x)$ and RNase inhibitor $(40 \mathrm{U} / \mu \mathrm{l})$ on ice for the duration of $30 \mathrm{~min}$. The cell lysates, pipetted up and down several times, were stored in $-80^{\circ} \mathrm{C}$ for $5 \mathrm{~min}$, then allowed to thaw on ice, and centrifuging at $12,000 \mathrm{~g}$ for $10 \mathrm{~min}$. Then the cell lysates were divided into two parts, one was saved as input group for the whole cell extraction, and the other was used for the following immunoprecipitation (IP) treatment (IP group). For the IP group, cell lysates were incubated with 5 $\mu$ g anti-IGF2BP2 (ab128175, Abcam, USA) or IgG antibody (14678-1-AP, Proteintech, Wuhan, China), respectively, which was alternated continually throughout the night at $4^{\circ} \mathrm{C}$. Protein $\mathrm{A} / \mathrm{G}$ magnetic beads (Bimake, China) were rinsed five consecutive times with $0.1 \%$ Tween-20 in PBS, and then mixed with cell lysate-antibody complexes and rotated continuously at $4^{\circ} \mathrm{C}$ for $6 \mathrm{~h}$. Next, the RNA-protein complexes were rinsed five consecutive times with elution buffer (containing $150 \mathrm{mM} \mathrm{NaCl}, 50 \mathrm{mM}$ Tris-HCl, $5 \mathrm{mM}$ EDTA, $0.5 \mathrm{mM}$ DTT, 0.5\% NP-40, 10\% SDS, and RNase inhibitor) and treated with proteinase $\mathrm{K}$ at $55^{\circ} \mathrm{C}$ for $1 \mathrm{~h}$. Bound RNAs were extracted and subjected to RT-qPCR for quantitative analysis. Relative enrichment was normalized to the input. The primers for RT-qPCR were showed in Additional file 1: Table S1.

\section{MeRIP-qPCR}

Total RNA was extracted as described above. Ten percent of the total RNA was reserved for the input control and the remaining RNAs were used for m6A-IP. Anti-m6A antibody (ab151230, abcam, USA) or mouse IgG were immobilized on magnetic beads using the Dynabeads ${ }^{T M}$ Antibody Coupling Kit (14311D, Invitrogen, USA) according to the manufacturer's instruction. Subsequently, total RNA was incubated with antibody-conjugated beads in 500 $\mu$ l binding buffer (containing $140 \mathrm{mM} \mathrm{NaCl}, 50 \mathrm{mM}$ Tris-HCl, $5 \mathrm{mM}$ EDTA, $0.5 \%$ NP-40, and RNase inhibitor) and rotated continually for $4 \mathrm{~h}$ at $4^{\circ} \mathrm{C}$. M6A-modified mRNAs were eluted from the beads with elution buffer and then purification was carried out for further analysis by RT-qPCR. Relative enrichment was normalized to the input. The primers for RT-qPCR were showed in Additional file 1: Table S1.

\section{Luciferase reporter assay}

$2 \times 10^{5} \mathrm{HNSCC}$ cells were first seeded in plates with 24 -wells, followed by culturing for $24 \mathrm{~h}$. Then plasmids carrying wild-type or mutated-type Slug CDS were transfected into the cells. After transfection for $12 \mathrm{~h}$, cells were re-seeded into a plate with 96 -wells and then for $24 \mathrm{~h}$ incubated. The Dual-Luciferase ${ }^{\circledR}$ Reporter Assay System (E1910, Promega, USA) was used to analyze the luciferase activities. Renilla Luciferase (Rluc) was used to achieve normalization of the firefly luciferase (F-luc) activity.

\section{mRNA stability assay}


HNSCC cells were seeded in plates with six-wells and grown to approximately $50 \%$ confluence following incubation for $24 \mathrm{~h}$. Then, actinomycin D ( $5 \mu \mathrm{g} / \mathrm{ml}$, Sigma, USA) was used to treat the cells, which were harvested at $0 \mathrm{~h}, 3 \mathrm{~h}$, and $6 \mathrm{~h}$. The total RNA was derived and then analyzed with qRT-PCR. The mRNA value of each group was calculated and normalized to GAPDH at the designated time. The degradation rate of mRNA was approximated based on previously published protocols [45].

\section{Statistical Analysis}

All the statistical analysis were conducted with GraphPad Prism (version 7.0, GraphPad software, USA) and SPSS 21.0 software (IBM, SPSS Statistics, USA). The correlation between IGF2BP2 expression and clinicopathologic parameters were analyzed with the Chi-Square test. Univariate and multivariate regression analyses were employed with the Cox proportional hazards model to identify independent factors affecting the survival of HNSCC patients. The Kaplan-Meier method was carried out to produce survival curves, and any significant differences in survival probability between groups were compared by log-rank statistics. We applied the two-tailed Student's $t$ test to compare results between two different groups, and the one-way ANOVA was conducted for multiple comparisons. All the data are presented in the form of mean $\pm S D$ and are the result of no less than 3 independently performed experiments. Statistical significance was determined as values of $P<0.05$. ${ }^{*} P<0.05, * \star P<0.01, * \star * P<0.001$, $\star \star \star \star P<0.0001$, ns: not statistically significant.

\section{Results}

\section{Expression and clinical significance of m6A regulators in HNSCC}

We analyzed the expression profiles of 20 m6A-related regulators in 502 HNSCC and 44 normal tissues from the TCGA database in order to study the role of m6A modification in HNSCC. As shown in Figure $1 \mathrm{~A}$ and $B, 18$ of the 20 m6A regulatory genes were significantly upregulated in HNSCC tissues compared with normal tissues, including 6 "writers" (VIRMA, ZC3H13, METTL14, METTL3, WTAP, and RBM15), 2 "erasers" (FTO and ALKBH5), and 10 "readers" (IGF2BP2, IGF2BP1, IGF2BP3, YTHDF3, YTHDF2, YTHDC1, YTHDF1, HNRNPC, RBMX, and HNRNPA2B1). There was no statistically significant difference between the expression levels of YTHDC2 and RBM15B in HNSCC tissues and normal tissues. To further investigate the clinical association of the m6A-related regulators in HNSCC, we first analyzed the OS rate of the $20 \mathrm{~m} 6 \mathrm{~A}$ regulatory genes in pan-cancer using GEPIA2. Intriguingly, among all these m6A-related genes, only the higher expression of IGF2BP2 is associated with a lower survival probability in HNSCC patients (Figure 1C). The Kaplan-Meier survival analysis based on TCGA data also supported this result, suggesting a potential prognostic role of IGF2BP2 in HNSCC (Figure 1D). The multivariate cox regression analysis revealed that IGF2BP2 was an independent risk factor in HNSCC patients (Figure 1E). In addition, the pathological $\mathrm{N}$ stage was also a significantly independent prognostic factor, suggesting that lymphatic metastasis is closely related to a poor prognosis in HNSCC patients (Figure 1E). Furthermore, 
we constructed a nomogram in accordance with the results of the multivariate cox regression analysis, in which the three independent prognostic factors, including IGF2BP2, pathological $\mathrm{N}$ stage and age were combined to produce a clinically quantitative method for predicting the 1-, 3-, and 5-year survival probability of HNSCC patients (Figure 1F). Every patient would accumulate a number of points for each prognostic parameter, and the more higher the total number of points, the poorer the outcome is for that particular patient [46]. The results of the calibration curve indicated a sufficient efficiency in estimating the 1-, 3-, and 5-year survival probability by using the nomogram (Figure 1G). Collectively, m6A regulators were commonly overexpressed in HNSCC, and IGF2BP2 was identified as a potential prognostic biomarker in HNSCC. Therefore, we focused on IGF2BP2 for further investigation.

\section{High expression of IGF2BP2 in HNSCC tissues and related to lymphatic metastasis}

We initially examined IGF2BP2's expression in HNSCC and NATs with RT-qPCR and western blot to evaluate its role in HNSCC. The RT-qPCR analysis demonstrated that IGF2BP2 was significantly overexpressed in HNSCC tumor samples compared with NATs (Figure 2A). Similar results were observed with western blot analysis in 5 cases of HNSCC tumor and NATs (Figure 2B). Our previous work has shown that cervical LN metastasis was correlated with the poor prognosis of HNSCC patients and was an independent risk factor for survival [47]. According to the results of the Kaplan-Meier survival analysis in Figure $1 \mathrm{C}$ and D, we wondered whether IGF2BP2 is involved in lymphatic metastasis, leading to a poor prognosis in HNSCC patients. To verify this hypothesis, 20 cases of HNSCC tissues with or without lymphatic metastasis (10 cases with lymphatic metastasis and 10 cases without lymphatic metastasis) were collected for assessing IGF2BP2 expression with RT-qPCR and western blot, respectively. Their results indicated that both the mRNA as well as protein levels of IGF2BP2 were increased in HNSCC tissues with lymphatic metastasis compared with those without lymphatic metastasis (Figure $2 \mathrm{C}$ and D). To further investigate the clinical relevance of IGF2BP2 in HNSCC, IHC was performed to detect IGF2BP2 expression in 78 cases of HNSCC patients, of which the complete clinicopathological characteristics and data on follow-up were available. Consistent with our data derived from RT-qPCR and western blotting, IHC analysis revealed that IGF2BP2 expression was marginally detected in NATs and slightly increased in HNSCC tissues without lymphatic metastasis but strongly upregulated in those with lymphatic metastasis (Figure 2E and F). Importantly, high IGF2BP2 expression was associated with poor OS probability (Figure $2 \mathrm{G}$ ). Moreover, analysis of the clinicopathological characteristics demonstrated that IGF2BP2 expression was significantly correlated with the pathological $\mathrm{N}$ classification and LN metastasis (Table 1). Univariate and multivariate Cox regression analyses demonstrated that IGF2BP2 expression was an independent prognostic factor in patients with HNSCC (Table 2). Collectively, these results imply that there is a high expression of IGF2BP2 in HNSCC and that it has an important role to play in LN metastasis. 


\section{IGF2BP2 promotes metastatic behaviour of HNSCC cells in vitro}

LN metastasis is a complex process involving multiple alterations, including an enhanced ability in cell migration and invasion $[48,49]$. To clarify whether IGF2BP2 induces the migration and invasion ability of HNSCC cells, FaDu and SCC15 HNSCC cell lines were transfected with three small interference sequences targeting different sites (si-IGF2BP2\#1, si-IGF2BP2\#2, and si-IGF2BP2\#3) of IGF2BP2 or were established to stably overexpress IGF2BP2 by lentivirus. The efficiency of knockdown and overexpression was validated by RT-qPCR and western blot, and the results indicated that IGF2BP2 was successfully silenced (si-IGF2BP2\#2 and si-IGF2BP2\#3) or overexpressed at the mRNA as well as protein levels in FaDu and SCC15 cells (Figure $3 A$ and B). Moreover, a wound-healing analysis demonstrated that knockdown of IGF2BP2 inhibited the migratory ability of FaDu and SCC15 cells, whereas the opposite results were observed after IGF2BP2 overexpression (Figure 3C and D). Transwell analysis further confirmed these results by showing that silencing IGF2BP2 attenuated the mobility and invasiveness of FaDu and SCC15 cells, whereas IGF2BP2 overexpression enhanced the cell metastasis. Collectively, these data indicate that IGF2BP2 promotes the migration and invasiveness of HNSCC cells.

\section{IGF2BP2 knockdown suppresses lymphatic metastasis and lymphangiogenesis in vivo}

To further determine the role of IGF2BP2 in lymphatic metastasis of HNSCC, we used nude mice to establish a popliteal LN metastasis model (Figure 4A), as mentioned by a previous study [50]. In brief, FaDu cells were transfected with lentiviral vector expressing shRNA and firefly luciferase to stably silence IGF2BP2 expression, and the silencing efficiency of IGF2BP2 was verified by RT-qPCR and western blot (Figure $4 \mathrm{~B}$ and $\mathrm{C}$ ). Then these cells were inserted into the footpads of nude mice through injection. After 4 weeks, the impact of IGF2BP2 on lymphatic metastasis was determined by in vivo bioluminescence imaging. Strikingly, IGF2BP2 knockdown notably inhibited the lymphatic metastasis of HNSCC cells (Figure 4D). Moreover, the footpad tumors and the popliteal lymph nodes were both smaller and lighter in the sh-IGF2BP2 group than in the sh-NC group, suggesting that IGF2BP2 suppressed the tumorigenesis and lymphatic metastasis of HNSCC (Figure 4E-J). In addition, the sh-IGF2BP2 group exhibited a lower $\mathrm{LN}$ metastatic rate than the sh-NC group (Figure 4K). Importantly, IHC analysis showed that silencing IGF2BP2 significantly decreased the levels of microlymphatic vessel density (MLD) in both the intratumoral and peritumoral regions of mice tissues, as indicated by LYVE1-positive microvessels (Figure $4 \mathrm{~L}$ ). Collectively, these findings suggest that IGF2BP2 knockdown significantly inhibits lymphatic metastasis and lymphangiogenesis in vivo.

\section{IGF2BP2 regulates EMT program of HNSCC cells}


EMT is a critical step in the initiation of tumor metastasis [35]. To further investigate the mechanism behind the lymphatic metastasis mediated by IGF2BP2, we established a EMT cell model using TGF- $\beta$, a potent EMT inducer [51], to analyze what role IGF2BP2 plays in regulating EMT. First, $10 \mathrm{ng} / \mathrm{ml}$ TGF- $\beta$ was used to treat FaDu and SCC15 cells. After 72h treatment, we observed loss of cell-cell contact and a spindle-shape morphology characterized by mesenchymal cells (Figure 5A). Furthermore, immunofluorescent staining and confocal imaging analysis showed that FaDu and SCC 15 cells treated with TGF- $\beta$ formed extensive filopodia and lamellipodia, as indicated by white arrows, which enabled cells to acquire migratory and invasive capabilities (Figure 5B). Moreover, western blot analysis revealed that TGF- $\beta$ induced the reduction of E-Cadherin expression in both HNSCC cells, which is considered a hallmark of an EMT shift (Figure 5C). These data suggests that HNSCC cell lines treated with TGF- $\beta$ were in the process of EMT.

Subsequently, we determined the role of IGF2BP2 in HNSCC cells undergoing EMT. RT-qPCR and western blot analyses showed that IGF2BP2 depletion downregulated N-Cadherin and vimentin's expression levels, whereas that of E-Cadherin was upregulated at the mRNA as well as protein levels in FaDu and SCC 15 cells treated with TGF- $\beta$ (Figure 5D and E). Moreover, immunofluorescent staining and confocal imaging analysis further confirmed that silencing IGF2BP2 led to a loss of vimentin expression, concomitant with a retention of E-Cadherin on the cell surface in both HNSCC cells, suggesting IGF2BP2 may regulate the EMT program (Figure 5F). To further confirm the role of IGF2BP2 in EMT, we detected the EMT-related markers in FaDu and SCC15 cells transduced with IGF2BP2 and the corresponding control vector without TGF- $\beta$ treatment, and observed that E-Cadherin was indeed downregulated, whereas $\mathrm{N}$-Cadherin and vimentin were upregulated at both the mRNA as well as protein levels (Figure $5 \mathrm{G}$ and $\mathrm{H}$ ). Taken together, these findings suggest that IGF2BP2 has an essential function in regulating the EMT program in HNSCC cells.

\section{Slug is involved in IGF2BP2-regulated EMT in HNSCC cells}

Given that EMT is directly or indirectly moderated by multiple predominant transcription factors (TFs), such as Snail, Slug, ZEB1, and Twist [37, 38], we therefore searched for the possible TFs that are regulated by IGF2BP2. In the TCGA database for HNSCC, the spearman correlation analysis revealed the strongest positive correlation between Slug and IGF2BP2 in 502 HNSCC tissue samples compared to Snail, ZEB1, and Twist, indicating a potential positive regulatory mechanism between IGF2BP2 and Slug in HNSCC (Figure 6A). Moreover, significantly decreased expression levels of Slug from IGF2BP2 ${ }^{\text {high }}$

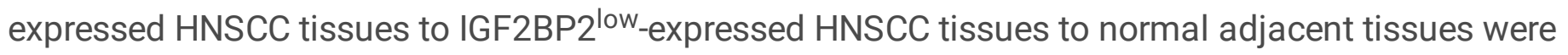
observed based on the TCGA database (Figure 6B). In order to clarify the regulatory relationship between IGF2BP2 and Slug, we evaluated the expression of Slug in HNSCC cells expressing different levels of IGF2BP2. RT-qPCR and western blot analyses revealed that IGF2BP2 inhibition significantly decreased Slug expression at mRNA as well as protein levels in FaDu and SCC15 cells, whereas overexpression of IGF2BP2 significantly enhanced Slug expression (Figure 6C-F). Meanwhile, there were no significant or consistent changes in the mRNA levels of Snail, ZEB1, and Twist in FaDu and SCC15 cells (Figure 6C). 
Although the roles of Slug in promoting EMT have been well studied $[39,43]$, we further investigated the roles of Slug in IGF2BP2-regulated EMT of cancer cells. The results of the western blot analysis indicated that Slug knockdown antagonized the upregulation of vimentin and downregulation of E-Cadherin induced by IGF2BP2 overexpression in FaDu cells (Figure 6G). Conversely, IGF2BP2 overexpression partially reversed vimentin's downregulation and E-Cadherin's upregulation caused by Slug inhibition in FaDu cells (Figure 6G). Furthermore, wound-healing assays showed that silencing Slug attenuated the promotion of cell migration via IGF2BP2 overexpression in FaDu cells (Figure 6H). Collectively, these findings indicate that Slug has a role in the IGF2BP2-regulated EMT in HNSCC cells.

\section{IGF2BP2 regulates slug mRNA stability via m6A modification}

Since IGF2BP2 is considered to be a RNA binding protein, also known as "reader" in m6A RNA modification $[8,9,19]$, we investigated whether IGF2BP2 interacts with Slug via m6A modification. First, we performed RIP-qPCR assays using the anti-IGF2BP2 antibody in FaDu and SCC15 cells, and the results showed significant enrichment of Slug mRNA compared to the IgG control group, confirming the interaction between IGF2BP2 and Slug mRNA (Figure 7A). In order to verify whether Slug is affected by m6A modification, leading to the recognition of the methylated Slug by IGF2BP2, we conducted MeRIPqPCR assays and determined that knockdown of IGF2BP2 markedly decreased the m6A levels of Slug in FaDu and SCC15 cells compared with the corresponding control cells (Figure 7B). Based on the SRAMP software analysis, we identified a very high-confidence m6A site in the CDS region upon Slug mRNA (Figure 7C). To validate the putative m6A site, we performed luciferase reporter assays using a luciferase reporter containing a wild-type (WT) Slug-CDS or mutated-type (MT) Slug-CDS sequence (GAACU to GACCU) (Figure 7D). As expected, the luciferase activity was significantly attenuated in Slug-WT when IGF2BP2 was silenced, while that of Slug-MT seemed to be unaffected (Figure 7E). Furthermore, mRNA stability assays revealed that the mRNA expression of Slug was decreased and the mRNA half-lives of Slug were continually reduced by IGF2BP2 silencing in FaDu and SCC15 cells (Figure 7F). In general, these findings suggest that IGF2BP2 directly binds the CDS region upon Slug mRNA in an m6A modification-dependent manner and stabilizes its mRNA.

\section{Discussion}

The primary cause of a poor prognosis in HNSCC patients is LN metastasis $[2,48]$. Although surgery is a reliable treatment in localized tumor in HNSCC [4], treatment options for metastatic HNSCC are currently limited. Thus, it is of utmost importance to investigate the potential molecular mechanisms involved in lymphatic metastasis, and to identify novel and efficient targets for therapeutic strategies. In this study, we identified IGF2BP2 as a potential prognostic marker in HNSCC. Moreover, IGF2BP2 overexpression in HNSCC cells was required in multiple steps of the lymphatic metastatic process, mainly including enhanced ability of cell motility and invasiveness, and formation of lymphatic vessels. In addition, IGF2BP2 regulated Slug expression, a key transcriptional factor of EMT, to facilitate the EMT program 
and metastasis of HNSCC cells. More importantly, we found that IGF2BP2 directly interacted with Slug mRNA and promoted its mRNA stability in an m6A-dependent manner (Figure 7G). These results offer novel insights related to the molecular mechanisms of cancer metastasis.

IGF2BP2, which acts as a novel m6A reader, is related to different biological processes, for instance, cell self-renewal, stemness maintenance, proliferation, and metastasis [10-12]. Previous studies have reported that IGF2BP2 is amplified and overexpressed in multiple cancers, and closely associated with clinical characteristics and poor prognosis $[15,52,53]$. The findings in this study have shown a high expression of IGF2BP2 in HNSCC tissues, and higher IGF2BP2 expression was significantly correlated to pathological N classification, LN metastasis and poor OS probability, indicating that IGF2BP2 could function as a prognostic biomarker in HNSCC, and plays a potential role in lymphatic metastasis of HNSCC. M6A modification has been reported to be associated with lymphatic metastasis in different cancers. Guo et al. [30] have found that the expression of the m6A reader HNRNPA2B1 is positively correlated with lymphatic metastasis, and promotes cell proliferation, migration, and invasion of ESCA. Wang et al. [31] have demonstrated that METTL3, a m6A writer, is closely associated with LN metastasis and a poor prognosis in cervical cancer. Nonetheless, which role IGF2BP2 plays in LN metastasis of HNSCC is still unknown. In the present study, it was shown that IGF2BP2 was highly expressed in HNSCC tissues with lymphatic metastasis compared with those without lymphatic metastasis, implying a potential role of IGF2BP2 in promoting lymphatic metastasis in cancers. To verify this, a popliteal LN metastasis model was constructed by injecting HNSCC cells into the footpad of nude mice, which is a sensitive and quantitative method of assessing lymphatic metastasis in vivo [54]. We found that IGF2BP2 knockdown could significantly inhibit lymphatic metastasis in vivo. Lymphangiogenesis is a rate-limiting step for the LN metastasis of cancer [55]. Consistently, we observed that silencing IGF2BP2 suppressed the formation of microlymphatic vessels in both the intratumoral and peritumoral regions of mouse tissues. Collectively, this study is the first to report on the discovery of the oncogenic role of IGF2BP2 in promoting lymphatic metastasis of HNSCC, indicating the broadly clinical significance of IGF2BP2 in cancer metastasis.

During the LN metastasis process, one important step is that tumor cells gain the capability of migration and invasion, and then move toward the lymphatic capillaries $[48,49]$. It has been established that EMT is a crucial step in metastasis and invasion of multiple cancers [35]. In this study, we found that IGF2BP2 could promote the EMT program to increase the mobility and invasiveness of HNSCC cells in vitro, which is in accordance with other studies $[52,56]$. The EMT process is controlled by a series of EMT-TFs, whose expression and importance are tissue-specific [37, 38]. Interestingly, among the EMT-TFs, such as Snail, Slug, ZEB1, and Twist, only Slug shows the strongest correlation with IGF2BP2 and is regulated by IGF2BP2 in HNSCC cell lines, thereby promoting the EMT program and cell metastasis. Slug, a conserved TF, plays an essential role in EMT during cancer metastasis [39, 40]. Puram et al. [57] investigated the intertumoral heterogeneity and metastasis between primary HNSCC tumors and matched LNs using single-cell transcriptomic analysis, and they found that Slug was the only EMT-related TF detected in HNSCC cells and closely correlated with the program across tumors. These discoveries align with our findings, further supporting the oncogenic role of IGF2BP2 in lymphatic metastasis of HNSCC cells. 
The biological importance of m6A modification is determined by $\mathrm{m} 6 \mathrm{~A}$ readers that can recognize and directly bind to the m6A modification sites upon its target mRNAs, thus affecting their fate [20,21]. To further investigate the regulatory mechanism between IGF2BP2 and Slug, we conducted RIP-qPCR analysis and determined that Slug is the direct target mRNA of IGF2BP2. Subsequently, MeRIP-qPCR analysis verified that IGF2BP2 regulated the mRNA expression of Slug through m6A modification. Furthermore, we predicted a very high-confidence m6A site in the CDS region upon Slug mRNA based on the SRAMP software analysis, which is a reliable and classic sequence-based m6A site predictor [58]. The results of the luciferase reporter analysis also confirmed this prediction. M6A readers have been linked to a variety of cellular processes, such as mRNA stability $[23,24,26]$. The Actinomycin D experiment confirmed that IGF2BP2 knockdown could significantly inhibit the mRNA stability of Slug. Similarly, Li et al. [59] demonstrated that IGF2BP2 can recognize and combine with the RNA methylation modification written by METTL3 to inhibit the degradation of SOX2, thereby promoting tumor progression. Hou et al. [60] revealed that IGF2BP2 cooperating with DHX9 enhances the HMGA1 mRNA stability by binding its 3'UTR and facilitates colorectal cancer proliferation and metastasis. Overall, these findings demonstrated that IGF2BP2 directly binds to the m6A site of the CDS region upon Slug mRNA and promotes the mRNA stability in a m6A-dependent manner, thereby facilitating EMT and lymphatic metastasis in HNSCC.

There are also some limitations in this study. First, the overall number of specimens is limited because access to tissue is invariably difficult. Second, although our findings reveal the oncogenic role of IGF2BP2 in promoting the lymphatic metastasis and EMT process, the regulatory mechanism between LN metastasis and EMT is not yet fully explained. This is probably because cancer cells undergoing EMT acquire the capacity to hijack a variety of chemotactic signals and apply them to disseminate through the lymphatic system [61]. However, a clearer understanding of the detailed mechanisms requires further investigation.

\section{Conclusions}

In summary, our study reveals for the first time the clinical and biological function of IGF2BP2 in facilitating lymphatic metastasis in HNSCC, and demonstrated that IGF2BP2 promotes EMT and cell metastasis via stabilizing Slug mRNA in an m6A dependent-manner. These outcomes indicate that IGF2BP2 could function as a predictive biomarker of $L N$ metastasis and potential target for antimetastasis therapies for HNSCC patients.

\section{Abbreviations}

HNSCC: Head and neck squamous carcinoma cells; LN: Lymph node; IGF2BP2: Insulin-like growth factor 2 mRNA-binding protein 2; RBP: RNA binding protein; m6A: N6-methyladenosine; YTHDF: YT521-B homology domain family; IGF2BPs: Insulin-like growth factor 2 mRNA-binding proteins; EMT: Epithelialmesenchymal transition; EMT-TFs: EMT transcriptional factors; CDS: Coding sequence; TCGA: The Cancer Genome Atlas; HR: Hazard ratio; Cl: Confidence interval; IHC: Immunohistochemistry; siRNA: Small interfering RNA; shRNA: Short hairpin RNA; MOI: Multiplicity of infection; qRT-PCR: Quantitative real-time 
PCR; RIP: RNA binding protein immunoprecipitation; MeRIP: Methylated RNA immunoprecipitation; OS: Overall survival; NAT: Normal adjacent tissue; MLD: microlymphatic vessel density; WT: Wild-type; MT: Mutated-type; T: tumor; LM (+): lymphatic metastasis; LM (-): non-lymphatic metastasis

\section{Declarations}

\section{Ethics approval and consent to participate}

The procedures were granted from the Ethical Review Committee of the First Affiliated Hospital of Chongqing Medical University.

\section{Consent for publication}

All authors approved this manuscript for publication in Journal of Experimental \& Clinical Cancer Research.

\section{Availability of data and material}

All data analyzed in this study are available from the corresponding author upon reasonable request.

\section{Competing interests}

No potential conflicts of interest.

\section{Funding}

This work was supported by the National Natural Science Foundation of China (82173303) and the Chongqing Joint Project of Science and Health (2020MSXM091).

\section{Authors' contributions}

GHH, DY and MP conceived and designed this study. DY performed the experiment with assistance from YSL and TL. DY wrote the manuscript. GHH and MP supervised the experiments and approved the manuscript. ZHW and CL provided assistance for patients' specimens collection. All authors approved the final version of manuscript. 


\section{Acknowledgements}

We sincerely thank to all team members for their assistance for this work.

\section{References}

1. Siegel RL, Miller KD, Jemal A. Cancer statistics. 2018. CA Cancer J Clin. 2018;68(1):7-30.

2. Johnson DE, Burtness B, Leemans CR, Lui VWY, Bauman JE, Grandis JR. Head and neck squamous cell carcinoma. Nat Rev Dis Primers. 2020;6(1):92.

3. Chow LQM. Head and Neck Cancer. N Engl J Med. 2020;382(1):60-72.

4. Galloway TJ, Ridge JA. Management of Squamous Cancer Metastatic to Cervical Nodes With an Unknown Primary Site. J Clin Oncol. 2015;33(29):3328-37.

5. Cramer JD, Burtness B, Le QT, Ferris RL. The changing therapeutic landscape of head and neck cancer. Nat Rev Clin Oncol. 2019;16(11):669-83.

6. Karam SD, Raben D. Radioimmunotherapy for the treatment of head and neck cancer. Lancet Oncol. 2019;20(8):e404-e16.

7. Ferris RL. Immunology and Immunotherapy of Head and Neck Cancer. J Clin Oncol. 2015;33(29):3293-304.

8. Degrauwe N, Suva ML, Janiszewska M, Riggi N, Stamenkovic I. IMPs: an RNA-binding protein family that provides a link between stem cell maintenance in normal development and cancer. Genes Dev. 2016;30(22):2459-74.

9. Liu HB, Muhammad T, Guo Y, Li MJ, Sha QQ, Zhang CX, et al. RNA-Binding Protein IGF2BP2/IMP2 is a Critical Maternal Activator in Early Zygotic Genome Activation. Adv Sci (Weinh). 2019;6(15):1900295.

10. Dai N. The Diverse Functions of IMP2/IGF2BP2 in Metabolism. Trends Endocrinol Metab. 2020;31(9):670-9.

11. Wang J, Chen L, Qiang P. The role of IGF2BP2, an m6A reader gene, in human metabolic diseases and cancers. Cancer Cell Int. 2021;21(1):99.

12. Cao J, Mu Q, Huang H. The Roles of Insulin-Like Growth Factor 2 mRNA-Binding Protein 2 in Cancer and Cancer Stem Cells. Stem Cells Int. 2018;2018:4217259.

13. Kim HY, Ha Thi HT, Hong S. IMP2 and IMP3 cooperate to promote the metastasis of triple-negative breast cancer through destabilization of progesterone receptor. Cancer Lett. 2018;415:30-9.

14. Li Z, Gilbert JA, Zhang Y, Zhang M, Qiu Q, Ramanujan K, et al. An HMGA2-IGF2BP2 axis regulates myoblast proliferation and myogenesis. Dev Cell. 2012;23(6):1176-88.

15. Xu X, Yu Y, Zong K, Lv P, Gu Y. Up-regulation of IGF2BP2 by multiple mechanisms in pancreatic cancer promotes cancer proliferation by activating the PI3K/Akt signaling pathway. J Exp Clin 
Cancer Res. 2019;38(1):497.

16. Dai N, Zhao L, Wrighting D, Kramer D, Majithia A, Wang Y, et al. IGF2BP2/IMP2-Deficient mice resist obesity through enhanced translation of Ucp1 mRNA and Other mRNAs encoding mitochondrial proteins. Cell Metab. 2015;21(4):609-21.

17. Kessler SM, Laggai S, Barghash A, Schultheiss CS, Lederer E, Artl M, et al. IMP2/p62 induces genomic instability and an aggressive hepatocellular carcinoma phenotype. Cell Death Dis. 2015;6:e1894.

18. Dai N, Ji F, Wright J, Minichiello L, Sadreyev R, Avruch J. IGF2 mRNA binding protein-2 is a tumor promoter that drives cancer proliferation through its client mRNAs IGF2 and HMGA1. Elife. 2017;6.

19. Huang H, Weng H, Sun W, Qin X, Shi H, Wu H, et al. Recognition of RNA N(6)-methyladenosine by IGF2BP proteins enhances mRNA stability and translation. Nat Cell Biol. 2018;20(3):285-95.

20. Roundtree IA, Evans ME, Pan T, He C. Dynamic RNA Modifications in Gene Expression Regulation. Cell. 2017;169(7):1187-200.

21. Zhao BS, Roundtree IA, He C. Post-transcriptional gene regulation by mRNA modifications. Nat Rev Mol Cell Biol. 2017;18(1):31-42.

22. Frye $M$, Harada BT, Behm M, He C. RNA modifications modulate gene expression during development. Science. 2018;361(6409):1346-9.

23. Zaccara S, Ries RJ, Jaffrey SR. Reading, writing and erasing mRNA methylation. Nat Rev Mol Cell Biol. 2019;20(10):608-24.

24. Shi H, Wei J, He C. Where, When, and How: Context-Dependent Functions of RNA Methylation Writers, Readers, and Erasers. Mol Cell. 2019;74(4):640-50.

25. Lee Y, Choe J, Park OH, Kim YK. Molecular Mechanisms Driving mRNA Degradation by m(6)A Modification. Trends Genet. 2020;36(3):177-88.

26. Zhao Y, Shi Y, Shen H, Xie W. m(6)A-binding proteins: the emerging crucial performers in epigenetics. J Hematol Oncol. 2020;13(1):35.

27. Liao S, Sun H, Xu CYTH, Domain. A Family of N(6)-methyladenosine (m(6)A) Readers. Genomics Proteomics Bioinformatics. 2018;16(2):99-107.

28. Wang T, Kong S, Tao M, Ju S. The potential role of RNA N6-methyladenosine in Cancer progression. Mol Cancer. 2020;19(1):88.

29. Zhao W, Qi X, Liu L, Ma S, Liu J, Wu J. Epigenetic Regulation of m(6)A Modifications in Human Cancer. Mol Ther Nucleic Acids. 2020;19:405-12.

30. Guo H, Wang B, Xu K, Nie L, Fu Y, Wang Z, et al. m(6)A Reader HNRNPA2B1 Promotes Esophageal Cancer Progression via Up-Regulation of ACLY and ACC1. Front Oncol. 2020;10:553045.

31. Wang Q, Guo X, Li L, Gao Z, Su X, Ji M, et al. N(6)-methyladenosine METTL3 promotes cervical cancer tumorigenesis and Warburg effect through YTHDF1/HK2 modification. Cell Death Dis. 2020;11(10):911. 
32. Zhang C, Hu J, Li H, Ma H, Othmane B, Ren W, et al. Emerging Biomarkers for Predicting Bladder Cancer Lymph Node Metastasis. Front Oncol. 2021;11:648968.

33. Eccles SA, Welch DR. Metastasis: recent discoveries and novel treatment strategies. Lancet. 2007;369(9574):1742-57.

34. Wan L, Pantel K, Kang Y. Tumor metastasis: moving new biological insights into the clinic. Nat Med. 2013;19(11):1450-64.

35. Pastushenko I, Blanpain C. EMT Transition States during Tumor Progression and Metastasis. Trends Cell Biol. 2019;29(3):212-26.

36. Lamouille S, Xu J, Derynck R. Molecular mechanisms of epithelial-mesenchymal transition. Nat Rev Mol Cell Biol. 2014;15(3):178-96.

37. Goossens S, Vandamme N, Van Vlierberghe P, Berx G. EMT transcription factors in cancer development re-evaluated: Beyond EMT and MET. Biochim Biophys Acta Rev Cancer. 2017;1868(2):584-91.

38. Stemmler MP, Eccles RL, Brabletz S, Brabletz T. Non-redundant functions of EMT transcription factors. Nat Cell Biol. 2019;21(1):102-12.

39. Cobaleda C, Perez-Caro M, Vicente-Duenas C, Sanchez-Garcia I. Function of the zinc-finger transcription factor SNAI2 in cancer and development. Annu Rev Genet. 2007;41:41-61.

40. Zhou W, Gross KM, Kuperwasser C. Molecular regulation of Snai2 in development and disease. J Cell Sci. 2019;132(23).

41. D'Uva G, Bertoni S, Lauriola M, De Carolis S, Pacilli A, D'Anello L, et al. Beta-catenin/HuR posttranscriptional machinery governs cancer stem cell features in response to hypoxia. PLoS One. 2013;8(11):e80742.

42. Jung CH, Kim J, Park JK, Hwang SG, Moon SK, Kim WJ, et al. Mdm2 increases cellular invasiveness by binding to and stabilizing the Slug mRNA. Cancer Lett. 2013;335(2):270-7.

43. Recouvreux MV, Moldenhauer MR, Galenkamp KMO, Jung M, James B, Zhang Y, et al. Glutamine depletion regulates Slug to promote EMT and metastasis in pancreatic cancer. J Exp Med. 2020;217(9).

44. Yu D, An X, Fan W, Wang X, He Y, Li B. PNUTS mediates ionizing radiation-induced CNE-2 nasopharyngeal carcinoma cell migration, invasion, and epithelial-mesenchymal transition via the PI3K/AKT signaling pathway. Onco Targets Ther. 2019;12:1205-14.

45. Liu J, Eckert MA, Harada BT, Liu SM, Lu Z, Yu K, et al. m(6)A mRNA methylation regulates AKT activity to promote the proliferation and tumorigenicity of endometrial cancer. Nat Cell Biol. 2018;20(9):1074-83.

46. Wang Y, Yang Z. A Gleason score-related outcome model for human prostate cancer: a comprehensive study based on weighted gene co-expression network analysis. Cancer Cell Int. 2020;20:159. 
47. Li Y, Lu T, Hu G. Gene sequencing and expression of Raf-1 in lymphatic metastasis of hypopharyngeal carcinoma. Cancer Biomark. 2020;28(2):181-91.

48. Achen MG, McColl BK, Stacker SA. Focus on lymphangiogenesis in tumor metastasis. Cancer Cell. 2005;7(2):121-7.

49. Nathanson SD. Insights into the mechanisms of lymph node metastasis. Cancer. 2003;98(2):41323.

50. Liu L, Lin C, Liang W, Wu S, Liu A, Wu J, et al. TBL1XR1 promotes lymphangiogenesis and lymphatic metastasis in esophageal squamous cell carcinoma. Gut. 2015;64(1):26-36.

51. Xu J, Lamouille S, Derynck R. TGF-beta-induced epithelial to mesenchymal transition. Cell Res. 2009;19(2):156-72.

52. Dahlem C, Barghash A, Puchas P, Haybaeck J, Kessler SM. The Insulin-Like Growth Factor 2 mRNA Binding Protein IMP2/IGF2BP2 is Overexpressed and Correlates with Poor Survival in Pancreatic Cancer. Int J Mol Sci. 2019;20(13).

53. Deng X, Jiang Q, Liu Z, Chen W. Clinical Significance of an m6A Reader Gene, IGF2BP2, in Head and Neck Squamous Cell Carcinoma. Front Mol Biosci. 2020;7:68.

54. Shah SA, Gallagher BM, Sands H. Lymphoscintigraphy of human colorectal carcinoma metastases in athymic mice by use of radioiodinated B72.3 monoclonal antibody. J Natl Cancer Inst. 1987;78(6):1069-77.

55. Stacker SA, Williams SP, Karnezis T, Shayan R, Fox SB, Achen MG. Lymphangiogenesis and lymphatic vessel remodelling in cancer. Nat Rev Cancer. 2014;14(3):159-72.

56. Mu Q, Wang L, Yu F, Gao H, Lei T, Li P, et al. Imp2 regulates GBM progression by activating IGF2/PI3K/Akt pathway. Cancer Biol Ther. 2015;16(4):623-33.

57. Puram SV, Tirosh I, Parikh AS, Patel AP, Yizhak K, Gillespie S, et al. Single-Cell Transcriptomic Analysis of Primary and Metastatic Tumor Ecosystems in Head and Neck Cancer. Cell. 2017;171(7):1611-24 e24.

58. Zhou Y, Zeng P, Li YH, Zhang Z, Cui Q. SRAMP: prediction of mammalian N6-methyladenosine (m6A) sites based on sequence-derived features. Nucleic Acids Res. 2016;44(10):e91.

59. Li T, Hu PS, Zuo Z, Lin JF, Li X, Wu QN, et al. METTL3 facilitates tumor progression via an m(6)AIGF2BP2-dependent mechanism in colorectal carcinoma. Mol Cancer. 2019;18(1):112.

60. Hou P, Meng S, Li M, Lin T, Chu S, Li Z, et al. LINC00460/DHX9/IGF2BP2 complex promotes colorectal cancer proliferation and metastasis by mediating HMGA1 mRNA stability depending on m6A modification. J Exp Clin Cancer Res. 2021;40(1):52.

61. Karlsson MC, Gonzalez SF, Welin J, Fuxe J. Epithelial-mesenchymal transition in cancer metastasis through the lymphatic system. Mol Oncol. 2017;11(7):781-91.

\section{Tables}

\section{Table 1}


Association between IGF2BP2 expression and clinicopathological characteristics of patients with HNSCC. 


\begin{tabular}{|c|c|c|c|c|c|}
\hline \multirow[t]{2}{*}{ Characteristics } & \multirow[t]{2}{*}{ Total } & \multicolumn{2}{|c|}{ IGF2BP2 expression } & \multirow[t]{2}{*}{$x^{2}$} & \multirow[t]{2}{*}{$P$ Valu } \\
\hline & & High & Low & & \\
\hline $\operatorname{Age}(\mathrm{y})$ & & & & 1.192 & 0.275 \\
\hline$\geq 60$ & 48 & 26 & 22 & & \\
\hline$<60$ & 30 & 20 & 10 & & \\
\hline Gender & & & & 0.448 & 0.504 \\
\hline male & 74 & 43 & 31 & & \\
\hline female & 4 & 3 & 1 & & \\
\hline T classification & & & & 1.184 & 0.757 \\
\hline $\mathrm{T} 1$ & 5 & 4 & 1 & & \\
\hline T2 & 13 & 8 & 5 & & \\
\hline T3 & 36 & 21 & 15 & & \\
\hline T4 & 24 & 13 & 11 & & \\
\hline $\mathrm{N}$ classification & & & & 8.804 & $0.032^{*}$ \\
\hline NO & 27 & 10 & 17 & & \\
\hline N1 & 10 & 6 & 4 & & \\
\hline N2 & 33 & 24 & 9 & & \\
\hline N3 & 8 & 6 & 2 & & \\
\hline Tumor Differentiation & & & & 2.096 & 0.351 \\
\hline well & 19 & 12 & 7 & & \\
\hline moderate & 39 & 20 & 19 & & \\
\hline poor & 20 & 14 & 6 & & \\
\hline Lymph node metastasis & & & & 8.214 & $0.004^{*}$ \\
\hline no & 27 & 10 & 17 & & \\
\hline yes & 51 & 36 & 15 & & \\
\hline Extranodal extension & & & & 0.115 & 0.734 \\
\hline no & 67 & 39 & 28 & & \\
\hline yes & 11 & 7 & 4 & & \\
\hline
\end{tabular}

Abbreviations: IGF2BP2: Insulin-like growth factor 2 mRNA-binding protein 2; HNSCC: Head and neck 
squamous carcinoma cells. The $P$ Value was measured by Chi-square test. ${ }^{\star} P<0.05$.

\section{Table 2}

Univariate analysis and multivariate analysis of IGF2BP2 expression and clinicopathologic variables in patients with HNSCC Cox-regression analysis.

\begin{tabular}{|c|c|c|c|c|}
\hline \multirow[t]{2}{*}{ Variables } & \multicolumn{2}{|l|}{ Univariate analysis } & \multicolumn{2}{|l|}{ Multivariate analysis } \\
\hline & $\mathrm{HR}(95 \% \mathrm{Cl})$ & $P$ Value & $\mathrm{HR}(95 \% \mathrm{Cl})$ & $P$ Value \\
\hline IGF2BP2 & $2.254(1.160-4.381)$ & $0.017^{\star}$ & $2.206(1.065-4.568)$ & $0.033^{*}$ \\
\hline Age & $1.006(0.545-1.857)$ & 0.983 & $1.154(0.594-2.244)$ & 0.672 \\
\hline Gender & $0.607(0.145-2.538)$ & 0.494 & $0.469(0.101-2.172)$ & 0.333 \\
\hline T classification & $1.157(0.554-2.416)$ & 0.698 & $1.087(0.496-2.382)$ & 0.836 \\
\hline Tumor differentiation & $1.164(0.608-2.226)$ & 0.647 & $1.049(0.528-2.084)$ & 0.892 \\
\hline LNM & 2.915 (1.295-6.559) & $0.010^{*}$ & $2.555(1.082-6.033)$ & $0.032^{\star}$ \\
\hline Extranodal extension & $0.704(0.296-1.678)$ & 0.429 & $0.978(0.065-1.568)$ & 0.653 \\
\hline \multicolumn{5}{|c|}{$\begin{array}{l}\text { Abbreviations: IGF2BP2: Insulin-like growth factor } 2 \text { mRNA-binding protein } 2 \text {; HNSCC: Head and neck } \\
\text { squamous carcinoma cells; HR: Hazard ratio; Cl: Confidence interval; LNM: Lymph node metastasis. } \\
\text { Variables: IGF2BP2: high vs low; Age: } \geq 60 \text { (y) vs<60 (y); Gender: male vs female; T classification: } \\
\text { T1/T2 vs T3/T4; Tumor differentiation: well/moderate vs poor; LNM: N0 vs N1/N2/N3; Extranodal } \\
\text { extension: no vs yes. }{ }^{*} P<0.05 \text {. }\end{array}$} \\
\hline
\end{tabular}

\section{Figures}


A

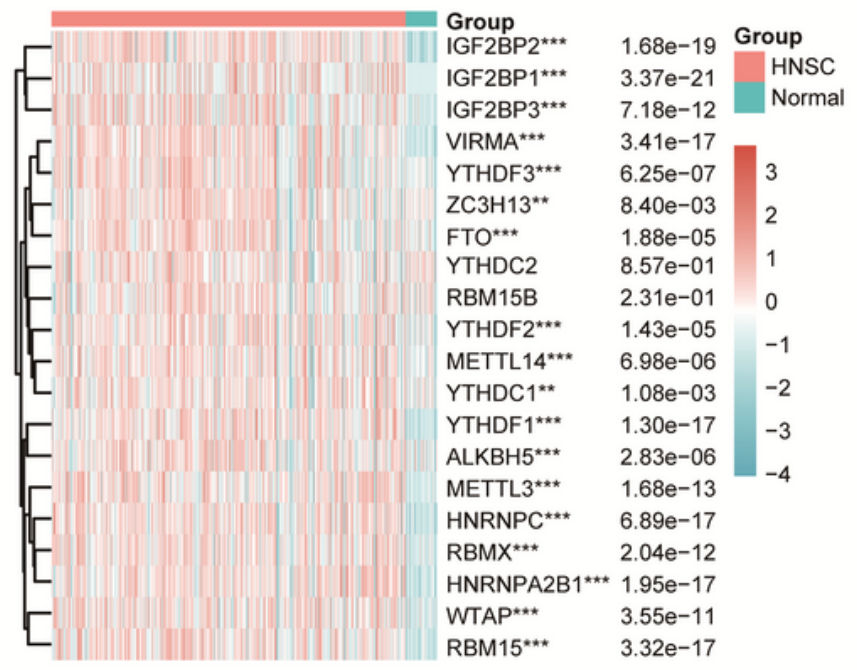

C

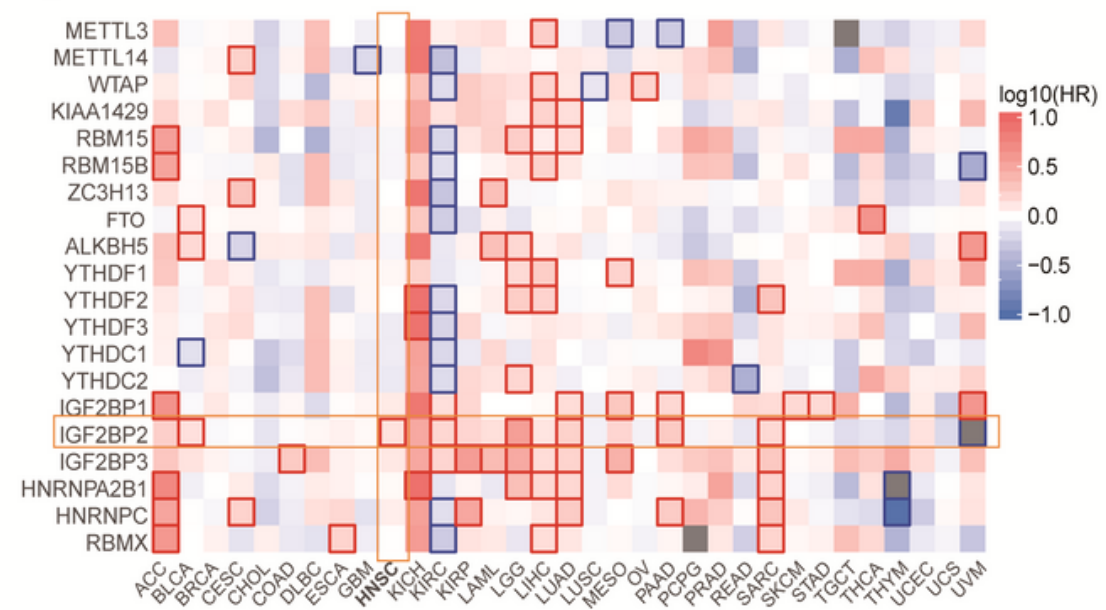

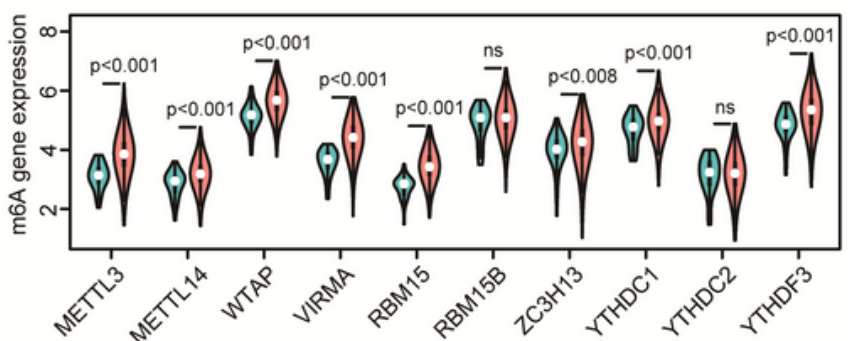

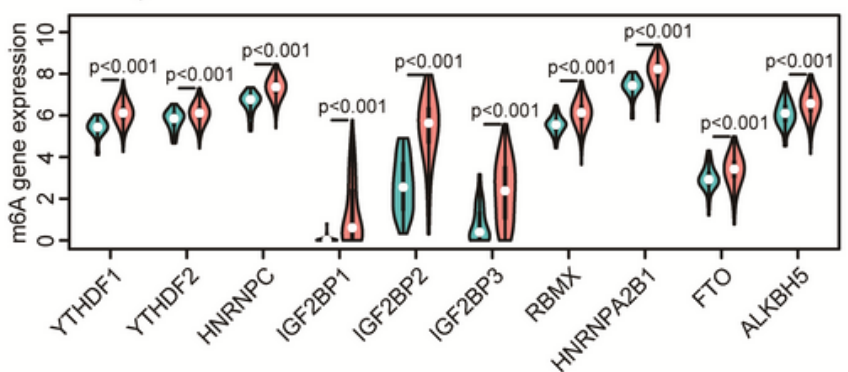

D
E

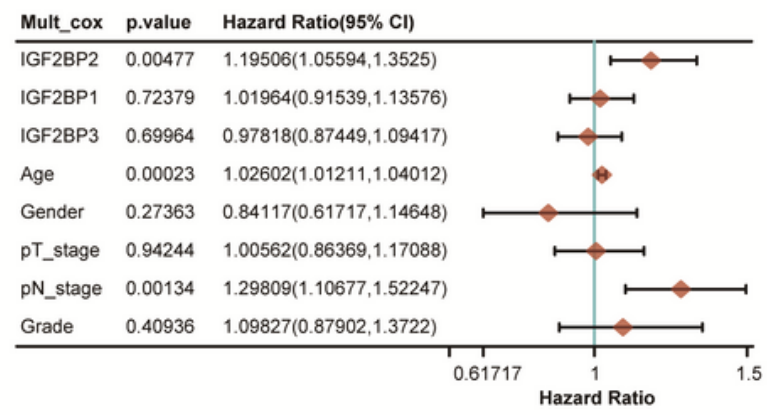

$\mathbf{F}$

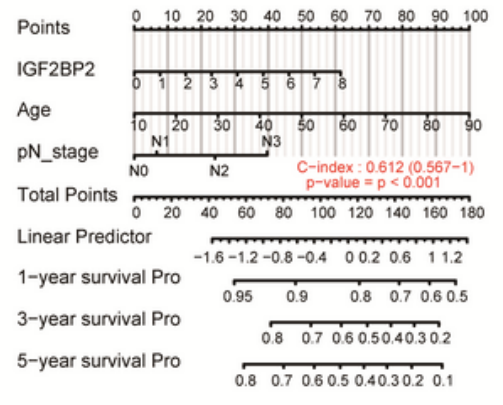

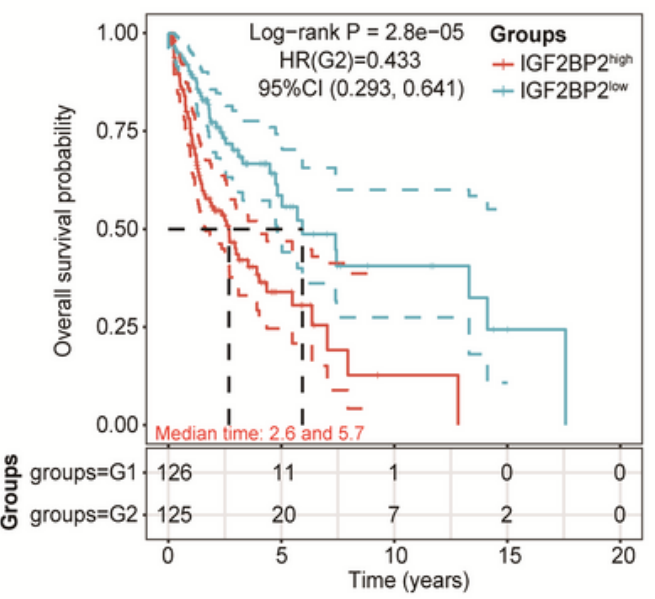

G

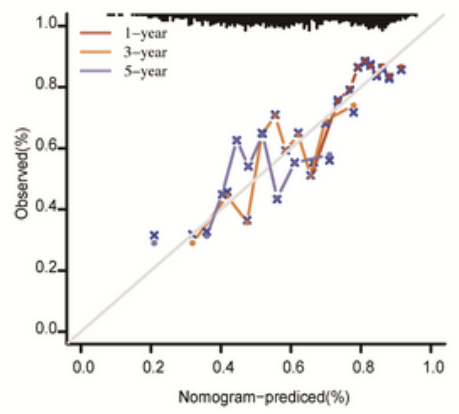

\section{Figure 1}

Expression and clinical significance of m6A regulators in HNSCC (A and $B$ ) Heatmap and violin plots show the mRNA expression levels of 20 m6A regulators in $502 \mathrm{HNSCC}$ and 44 normal tissues according to TCGA data. Red and blue colors illustrate relative high and low expression, respectively. ${ }^{\star \star} \mathrm{P}<0.01$, ${ }^{\star} * \star \mathrm{P}<0.001$, ns: not statistically significant. (C) The survival map of $20 \mathrm{~m} 6 \mathrm{~A}$-related regulators in pancancer generated by GEPIA2 online website. (D) The OS probability of IGF2BP2 for patients with HNSCC 
in two groups based on TCGA data. Group 1: Top 25\% HNSCC patients with high IGF2BP2 expression. Group 2: Top 25\% HNSCC patients with low IGF2BP2 expression. (E) Multivariate cox regression analysis of IGF2BPs and clinical parameters to identify the independent prognostic factors. (F) The nomogram for prediction of the 1-, 3-, and 5-year OS of HNSCC patients. (G) The calibration curve for the OS nomogram model. The ideal nomogram is represented by a dashed diagonal line, and the blue, red, and orange lines symbolize the 1-, 3-, and 5-year observed nomograms.

A

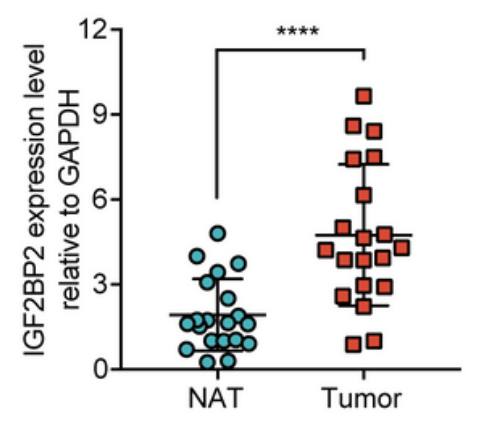

C

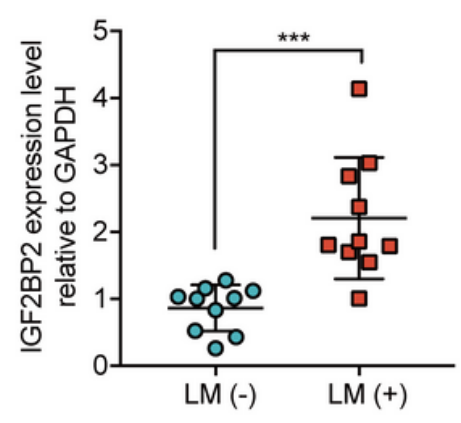

$E$

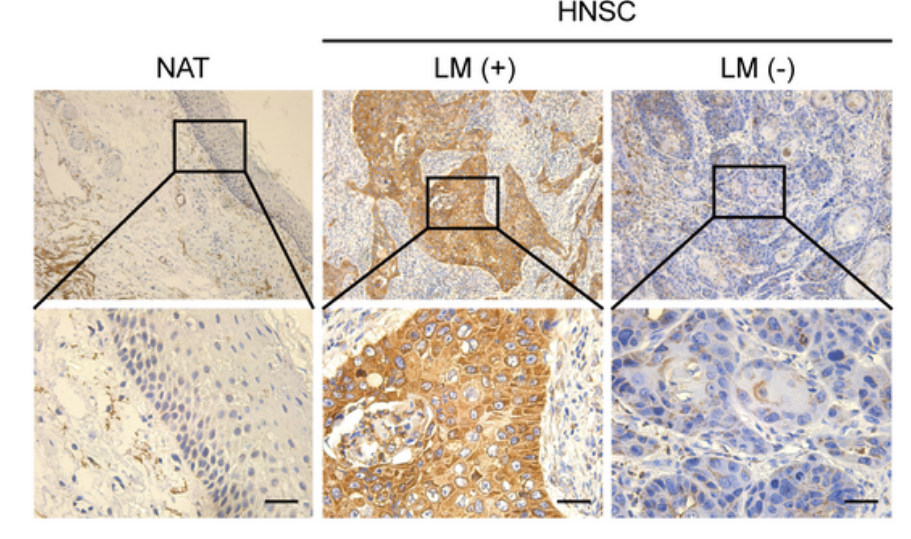

B

D
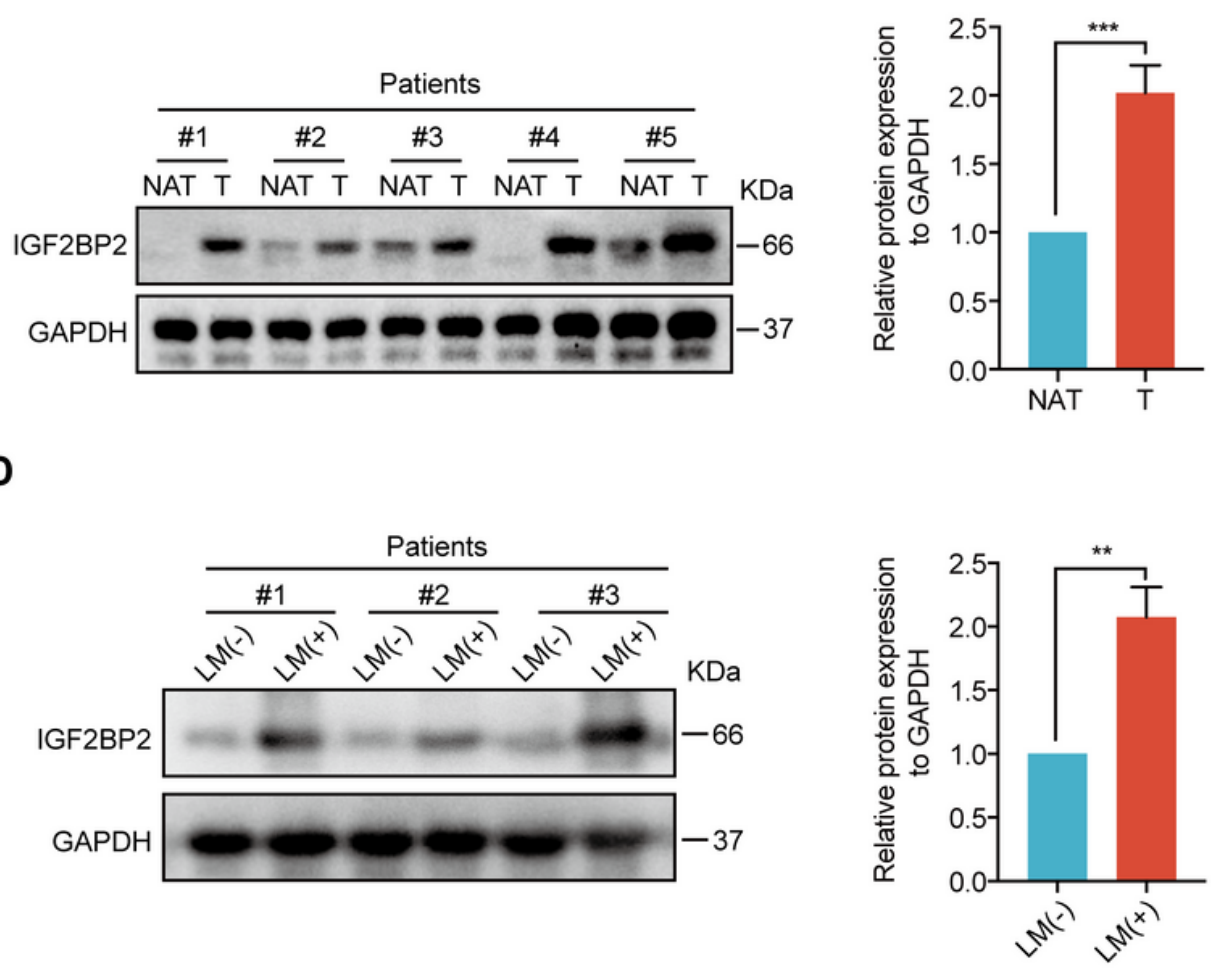

$\mathbf{F}$

G

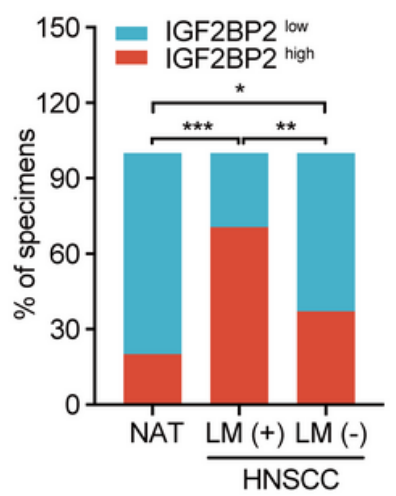

\section{Figure 2}

IGF2BP2 highly expressed in HNSCC tissues and related to lymphatic metastasis (A) RT-qPCR analysis of the expression of IGF2BP2 in HNSCC tissues $(n=20)$ and NATs $(n=20)$. NAT: normal adjacent tissues; T: tumor. ${ }^{* * *} P<0.0001$. (B) Western blot analysis of IGF2BP2 expression in HNSCC tissues and NATs $(n=$ 
5). Right panel shows the statistical data. ${ }^{\star} *{ }^{*}<0.001$. (C) RT-qPCR analysis of the expression of IGF2BP2 in HNSCC tissues with or without lymphatic metastasis $(n=20$, including 10 cases with lymphatic metastasis and 10 cases without lymphatic metastasis). LM (+): lymphatic metastasis; LM (-): nonlymphatic metastasis. ${ }^{\star \star *} \mathrm{P}<0.001$. (D) Western blot analysis of the expression of IGF2BP2 in HNSCC tissues with or without lymphatic metastasis. Statistical data is shown in the right panel. ${ }^{\star *} \mathrm{P}<0.01$. (E-F) Representative images (E) and percentages (F) of IGF2BP2 expression in the paraffin-embedded HNSCC tissues with or without lymphatic metastasis and NATs. Scale bars: $200 \mu \mathrm{m} .{ }^{*} P<0.05,{ }^{*} P<0.01$, $\star \star \star P<0.001$. (G) Kaplan-Meier curves of OS in HNSCC patients with high and low expression of IGF2BP2. The median IGF2BP2 expression was applied as the cutoff value. GAPDH served as an internal control.

A
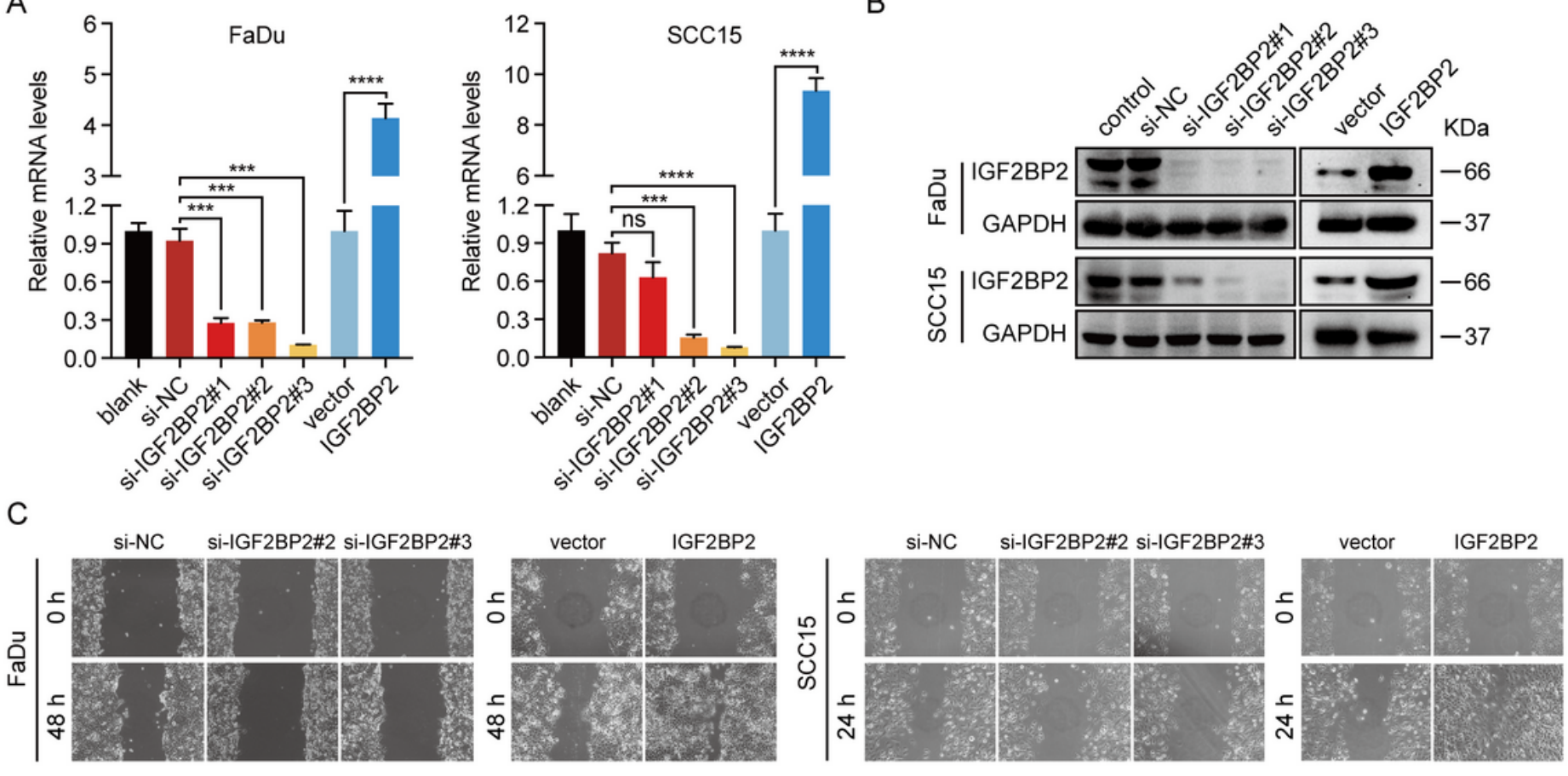

D
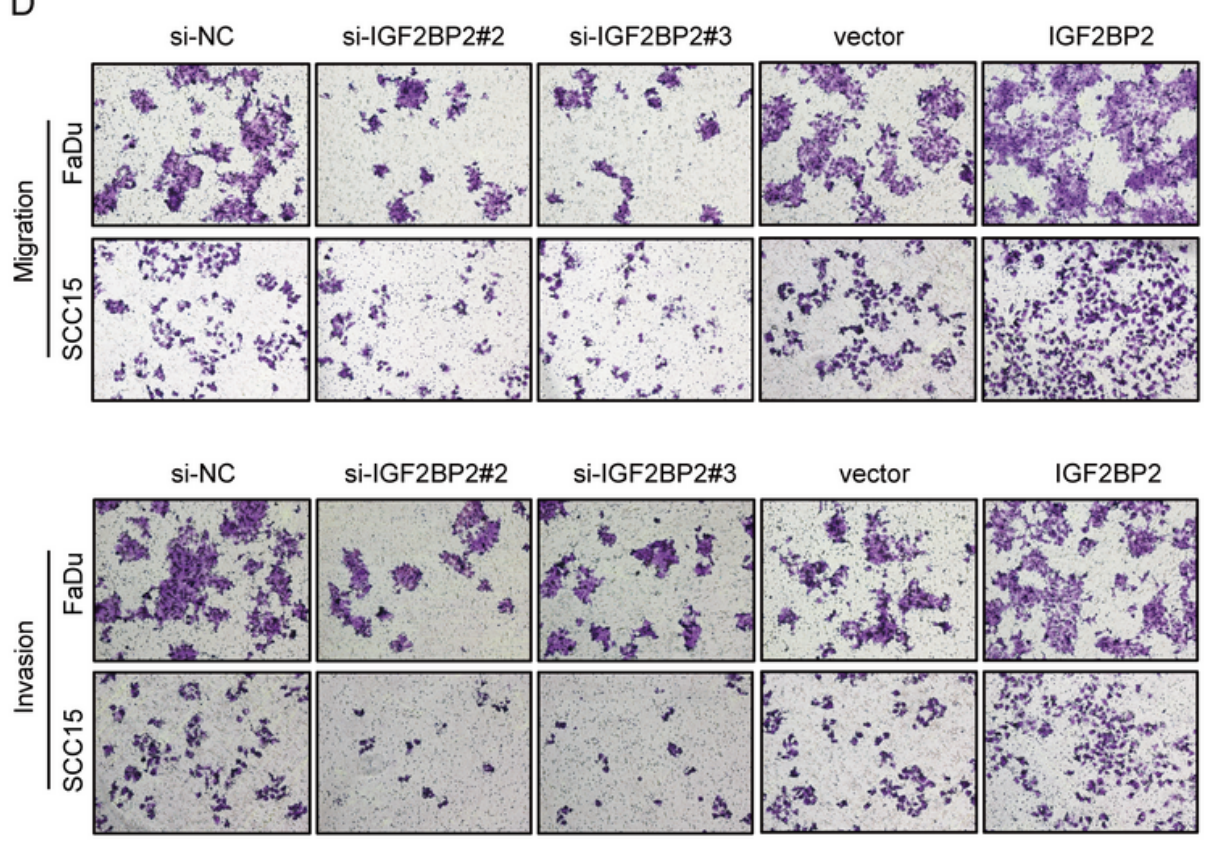

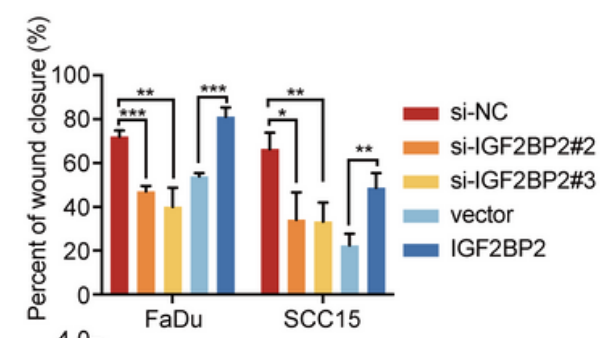

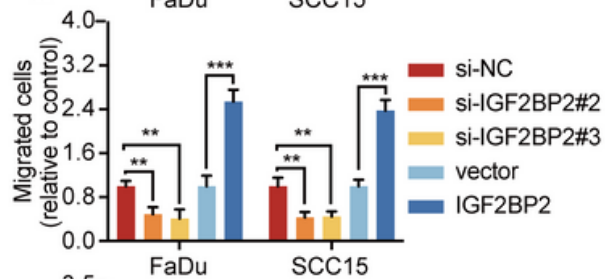

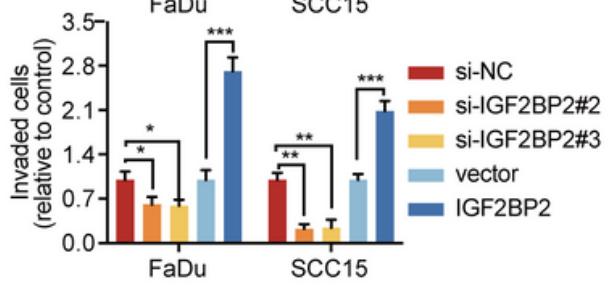




\section{Figure 3}

IGF2BP2 promotes metastatic behaviour of HNSCC cells in vitro (A-B) The efficiency of knockdown and overexpression of IGF2BP2 was validated by RT-qPCR (A) and western blot (B) at mRNA as well as protein levels in FaDu and SCC15 cells. GAPDH served as an internal control. ${ }^{\star \star *} P<0.001,{ }^{\star}{ }^{*} * \star P<0.0001$, ns: not statistically significant. (C-D) Images representing wound healing (C) and transwell assays (D) showing the mobility and invasiveness of HNSCC cells after knockdown or overexpression of IGF2BP2. Lower right panel shows the statistical data of cell migration distance, and migrated and invaded cell counts. ${ }^{*} P<0.05,{ }^{*} P<0.01, * * * P<0.001$. 
A

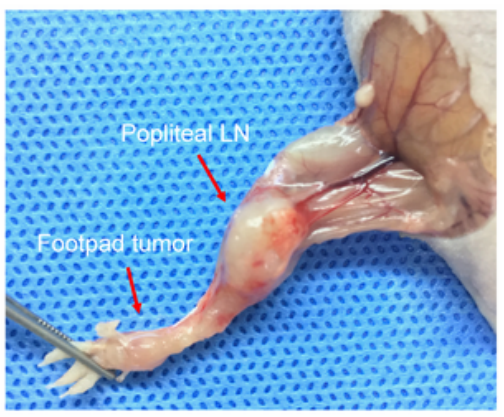

D
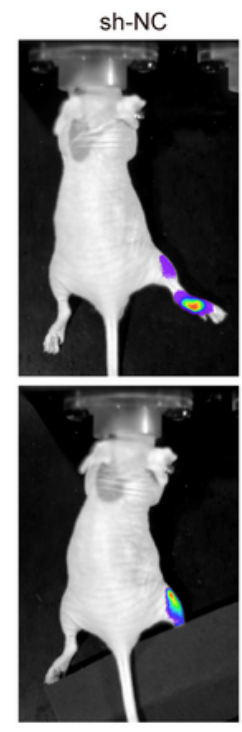

$\mathbf{L}$

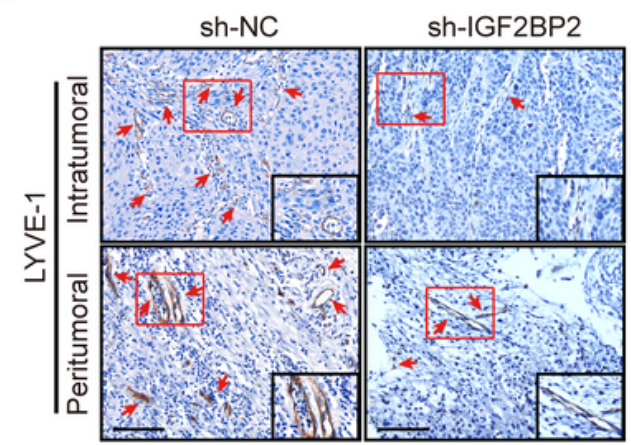

B

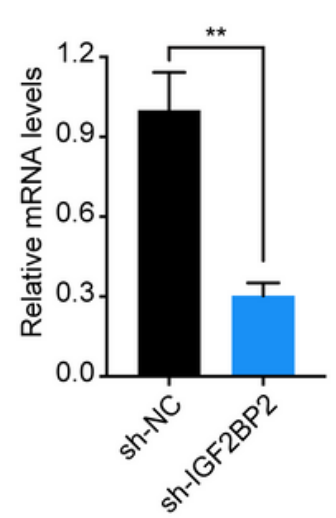

E
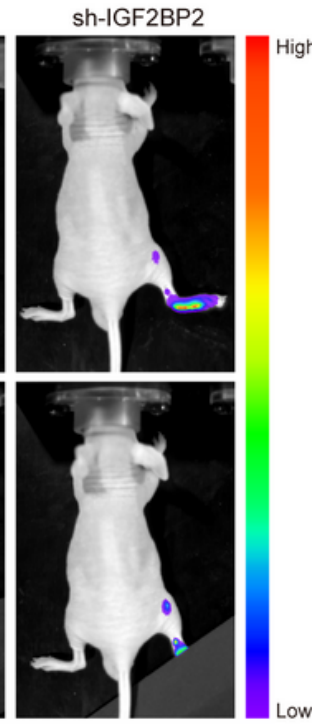

H

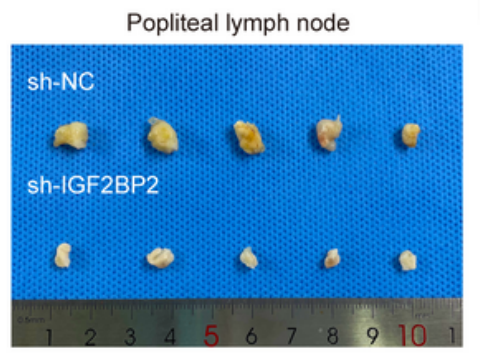

K

C

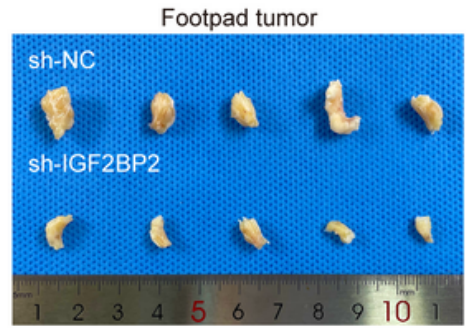

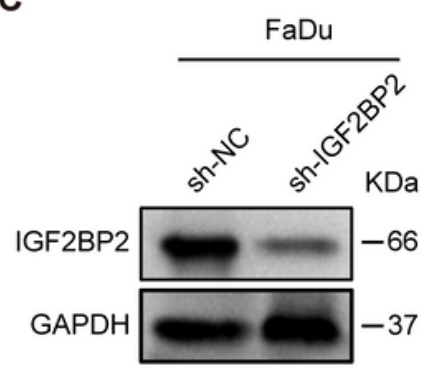

$\mathbf{F}$
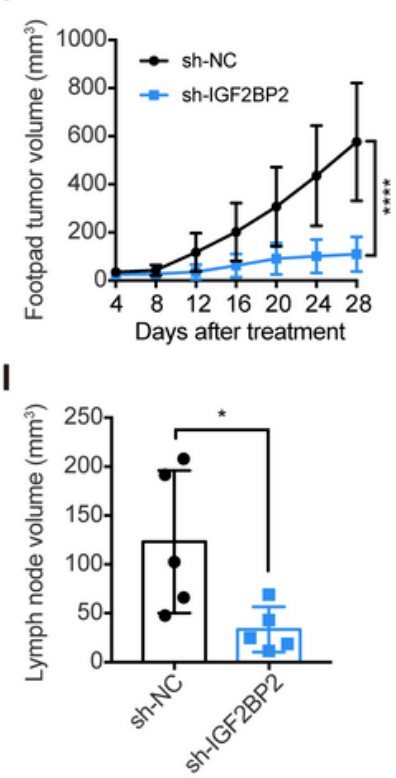

G
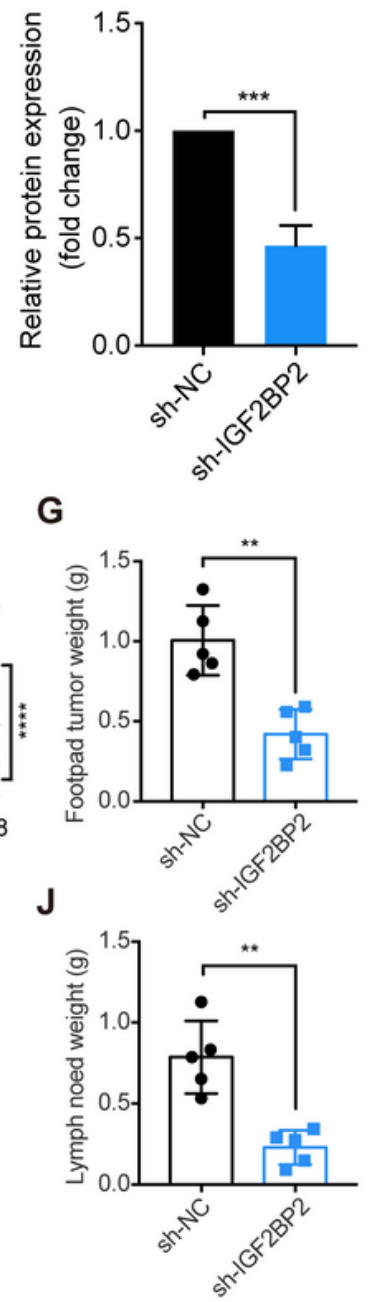

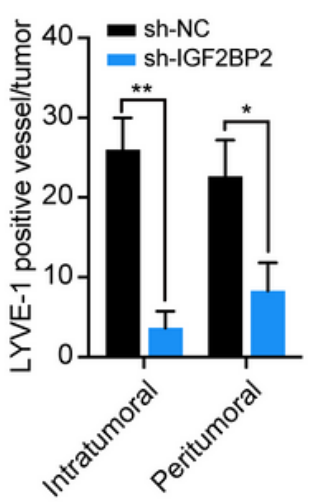

Effect of IGF2BP2 on popliteal LN metastasis in vivo.

\begin{tabular}{|c|c|c|c|c|}
\hline Groups & $\begin{array}{l}\text { No. } \\
\text { Metastasis } \\
\text { LNs }\end{array}$ & $\begin{array}{l}\text { No. } \\
\text { Non-metastasis } \\
\text { LNs }\end{array}$ & $\begin{array}{l}\text { Metastasis } \\
\text { ratio }\end{array}$ & $P$-Value ${ }^{a}$ \\
\hline sh-NC & 9 & 1 & $90 \%$ & \multirow[t]{2}{*}{$0.006^{* *}$} \\
\hline sh-IGF2BP2 & 3 & 7 & $30 \%$ & \\
\hline
\end{tabular}

${ }^{a}$ Chi-square test

\section{Figure 4}

IGF2BP2 knockdown suppresses lymphatic metastasis and lymphangiogenesis in vivo (A)

Representative images of the popliteal lymph node (LN) metastasis model in nude mice. FaDu cells were inserted into the footpads of nude mice through injection, and the metastasized popliteal LNs were harvested and analyzed. (B-C) RT-qPCR (B) and western blot (C) analysis of IGF2BP2 expression at mRNA as well as protein levels in stably IGF2BP2-silenced cells and control cells. Right panel shows the 
statistical analysis of western blotting. GAPDH functioned as internal control. ${ }^{\star *} P<0.01,{ }^{\star \star *} P<0.001$. (D) Representative images of bioluminescence of popliteal LN metastasis after inhibition of IGF2BP2 $(n=10$ per group). (E) Representative images of enucleated footpad tumors for the indicated groups ( $n=10$ per group). ( $F-G)$ The volume (F) and weight (G) of footpad tumors for the indicated groups. ${ }^{* * P}<0.01$, $\star \star \star \star P<0.0001$. $(H)$ Images representing enucleated popliteal $L N s$ for the indicated groups $(n=10$ per group). (I-J) The volume (I) and weight (J) of lymph nodes for the relevant groups. ${ }^{*} P<0.05,{ }^{*} P<0.01$. (K) The metastatic ratio of popliteal LNs was calculated for all groups $(n=10$ per group, $P=0.006)$. $(L)$ Representative images of mice tissues immunostained with anti-LYVE-1 antibody in intratumoral or peritumoral regions with different IGF2BP2 expression levels. Red arrows indicate the microlymphatic vessels. Right panel shows the quantification of microlymphatic vessel density. Scale bars: $200 \mu \mathrm{m}$. ${ }^{*} \mathrm{P}<0.05,{ }^{* *} \mathrm{P}<0.01$. 
A
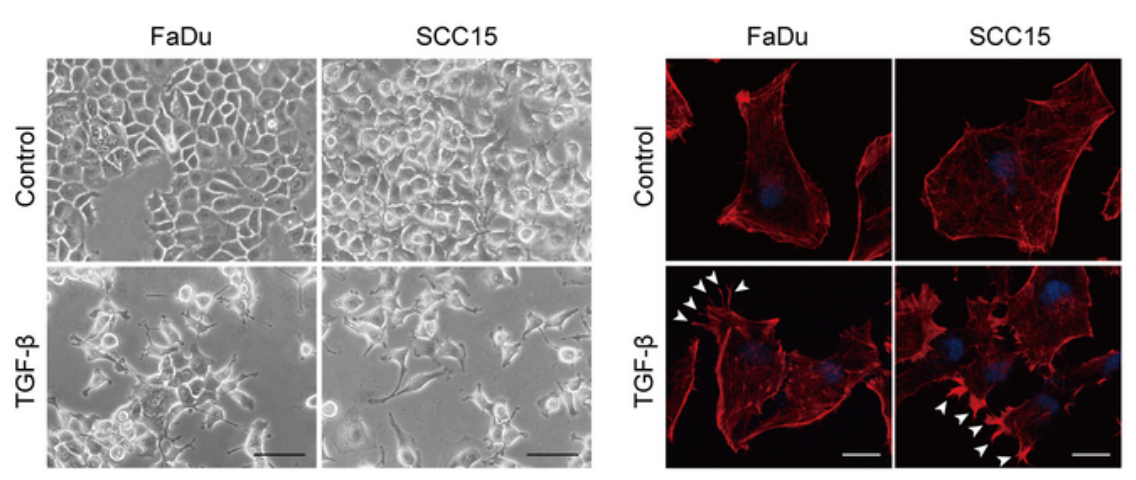

D

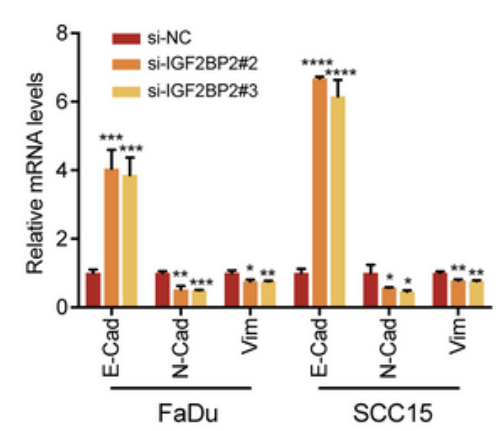

E

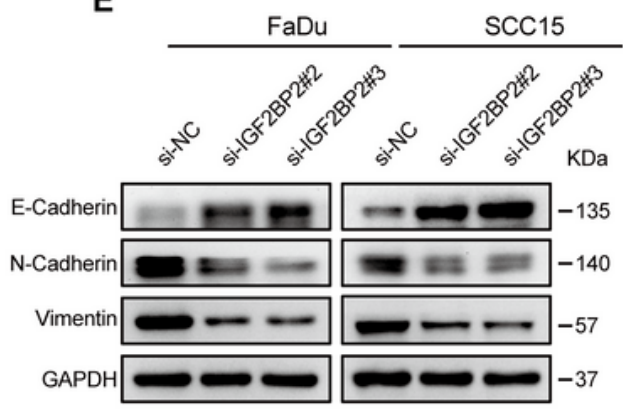

$\mathbf{F}$
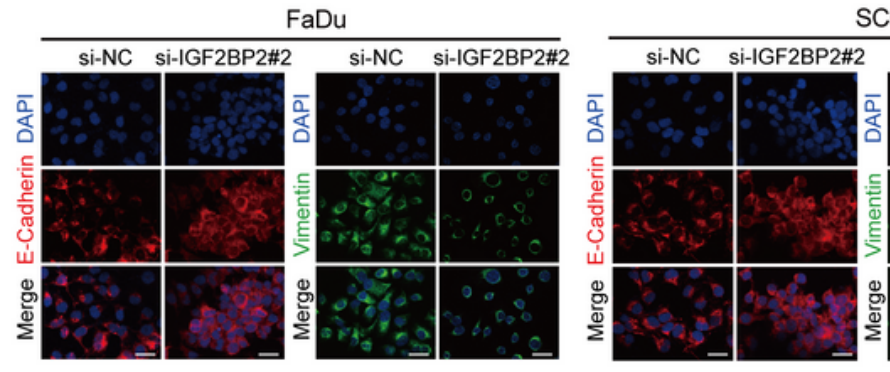

$\mathbf{H}$

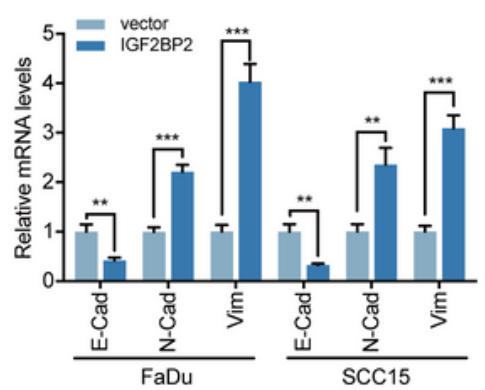

C
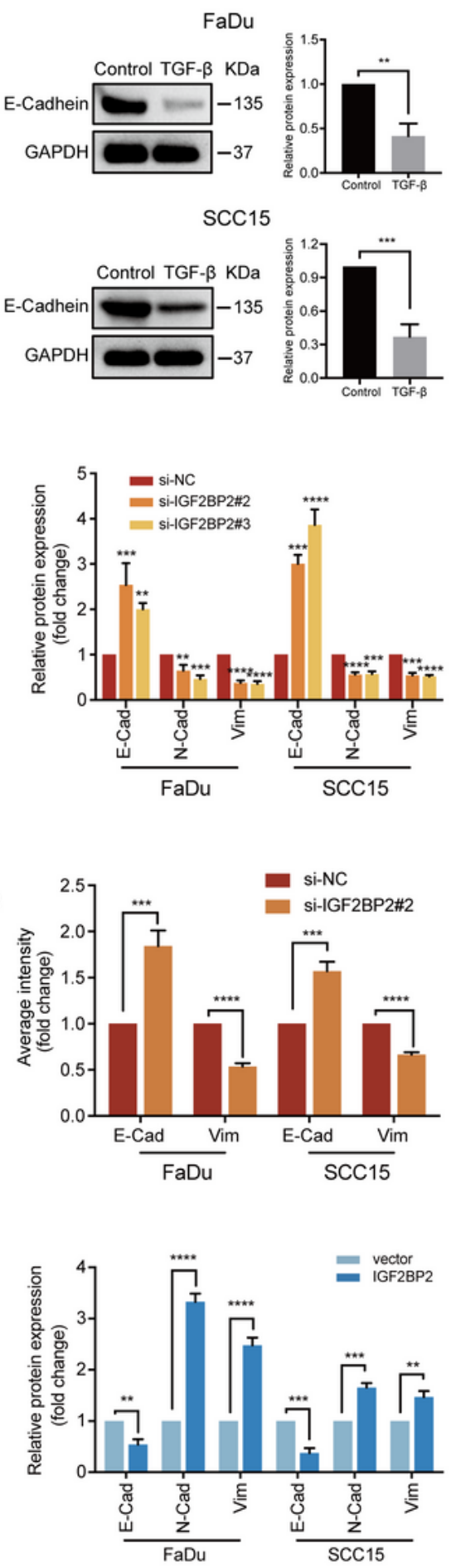

\section{Figure 5}

IGF2BP2 regulates EMT program of HNSCC cells (A) FaDu and SCC15 cells were either treated with TGF$\beta(10 \mathrm{ng} / \mathrm{ml})$ or without. Cell morphologic changes were observed after $72 \mathrm{~h}$. Representative images from 3 independent experiments are shown. Scale bars: $200 \mu \mathrm{m}$. (B) FaDu and SCC15 cells were either treated with TGF- $\beta$ or without. Cytoskeleton alterations were assessed by immunofluorescence staining with phalloidin, and by confocal scanning after $72 \mathrm{~h}$. White arrows indicate the extensive filopodia and 
lamellipodia. Representative images from 3 independent experiments are shown. Scale bars: $50 \mu \mathrm{m}$. (C) The results of the western blot analysis of the expression of E-Cadherin in FaDu and SCC15 cells either treated with TGF- $\beta$ or without. Right panel shows the statistical analysis of western blot. ${ }^{*} P<0.01$, $\star * * P<0.001$. (D-E) FaDu and SCC15 cells underwent transfection with si-RNAs or si-NC after TGF- $\beta$ treatment. The levels of E-Cadherin, N-Cadherin, and vimentin expression were identified with RT-qPCR (D) and western blotting (E). ${ }^{*} P<0.05,{ }^{*} P<0.01,{ }^{*} * \mathrm{P}<0.001$, ${ }^{\star \star \star *} \mathrm{P}<0.0001$. (F) FaDu and SCC15 cells were transfected with si-RNAs or si-NC after TGF- $\beta$ treatment. The expression and distribution of E-Cadherin and vimentin was identified by immunofluorescence and confocal scanning. Red: E-Cadherin; Green: vimentin; Blue (DAPI): nuclei. Scale bars: $100 \mu \mathrm{m}$. Right panel shows the statistical analysis of

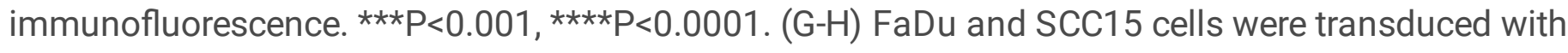
IGF2BP2 and a corresponding control vector, without TGF- $\beta$ treatment. The expression levels of ECadherin, N-Cadherin, and vimentin were identified with RT-qPCR $(G)$ and western blotting $(H)$. ${ }^{*} P<0.01$,

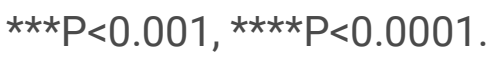



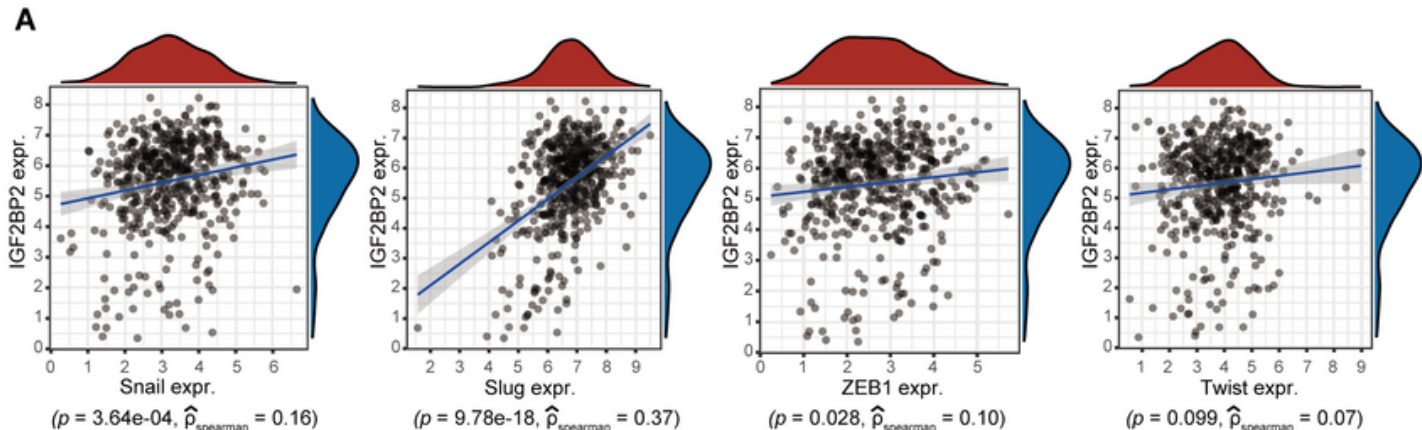

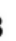

C
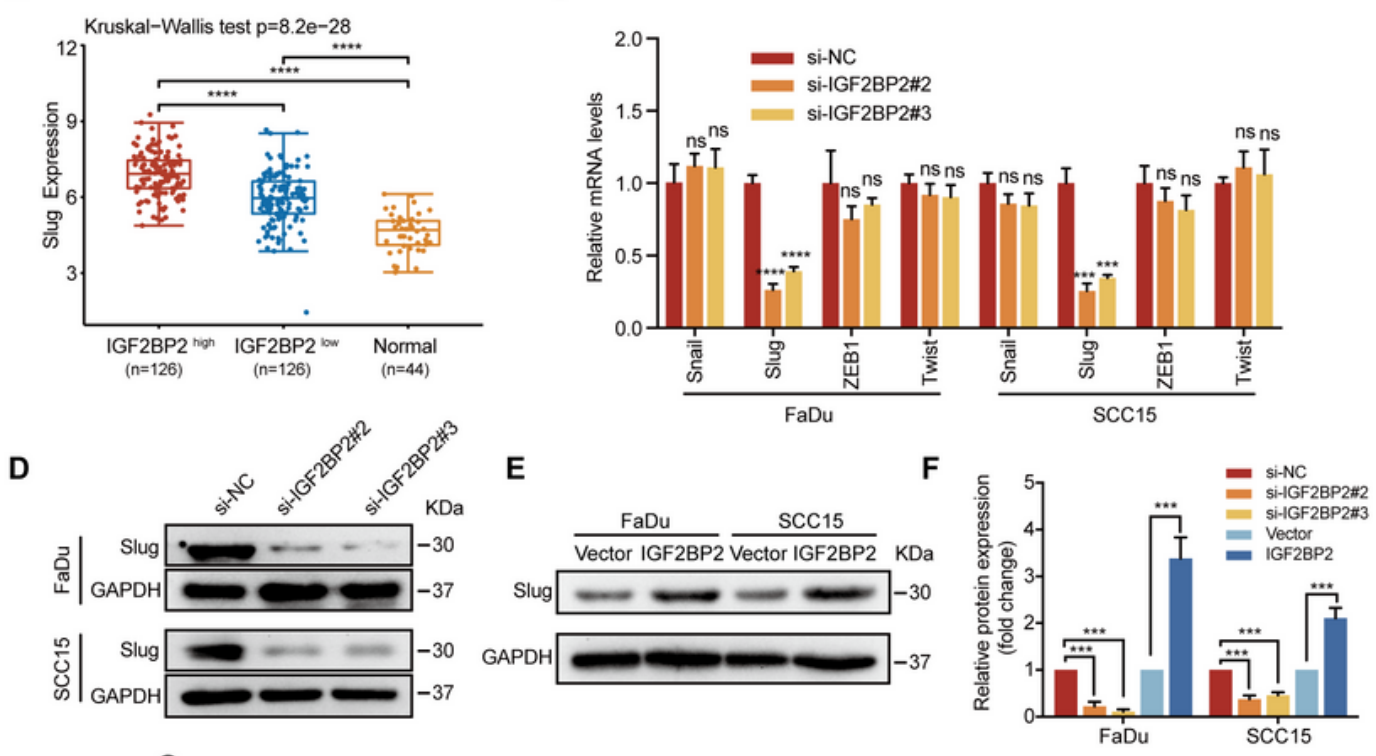

G

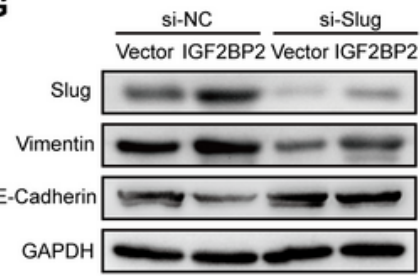

H
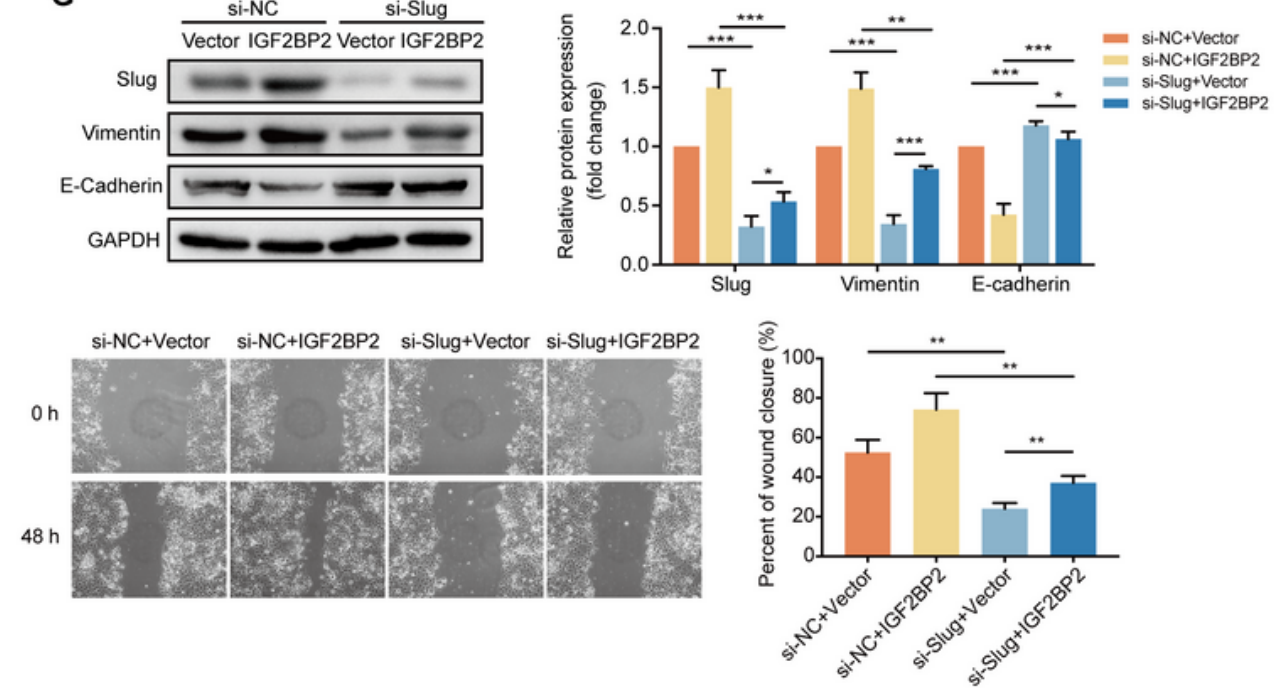

\section{Figure 6}

Slug is involved in IGF2BP2-regulated EMT in HNSCC cells (A) Spearman correlation of Snail, Slug, ZEB1, and Twist with IGF2BP2 in the TCGA database for HNSCC. P value and Spearman's correlations (rho) are indicated. (B) The distribution of Slug expression in HNSCC tissues with high or low IGF2BP2 expression and normal tissues. The uppermost corner on the left depicts the test method for significant $P$ values. (C) FaDu and SCC15 cells were transfected with si-RNAs or si-NC after TGF- $\beta$ treatment. The mRNA levels of 
Snail, Slug, ZEB1, and Twist were detected by RT-qPCR. ${ }^{*} * \mathrm{P}<0.001$, ${ }^{\star \star \star \star} P<0.0001$, ns: not statistically significant. (D) FaDu and SCC15 cells underwent transfection with si-RNAs or si-NC after TGF- $\beta$ treatment. The expression of Slug was detected by western blot analysis. (E) The expression of Slug was detected by western blotting analysis in IGF2BP2-overexpressed FaDu cells or corresponding control cells without TGF- $\beta$ treatment. (F) Statistical analysis of western blot for all the groups. ${ }^{* \star *} P<0.001$, $\star \star \star \star P<0.0001$. (G) IGF2BP2-overexpressed FaDu cells or corresponding control cells underwent transfection with si-NC or si-Slug for $48 \mathrm{~h}$, and the expression of Slug, vimentin, and E-Cadherin were assessed by western blot analysis (left) and analyzed quantitatively (right). ${ }^{*} P<0.05,{ }^{*} P<0.01$, $\star \star \star P<0.001$. (H) After transfection with si-NC or si-Slug for 48h, the wound healing of IGF2BP2overexpressed FaDu cells and corresponding control cells were recorded (left) and analyzed quantitatively (right). ${ }^{\star} * \mathrm{P}<0.01$. 
A
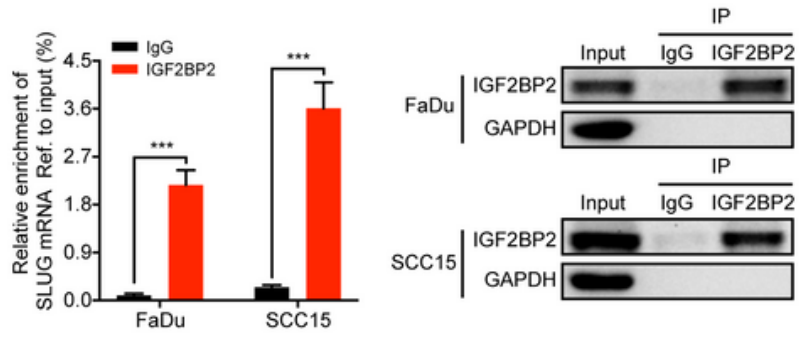

C

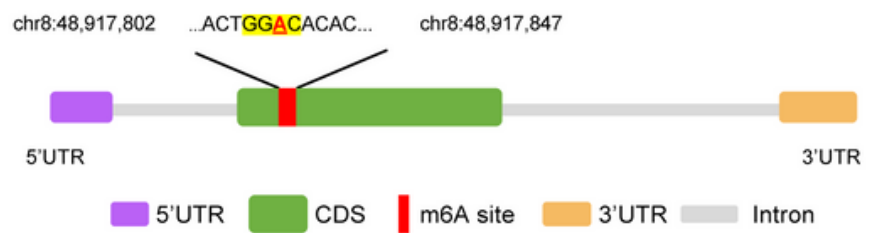

D

Slug-CDS WT ...AACTACAGCGAACTGGACACACATACAGGTA.. Slug-CDS Mut ..AACTACAGCGAACTGGICACACATACAGGTA...
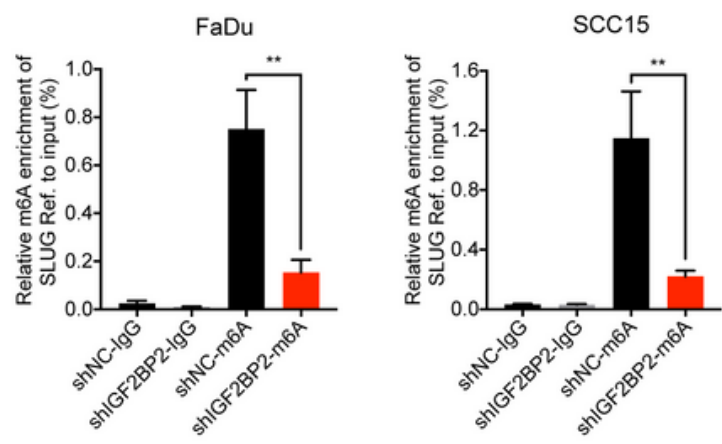

E
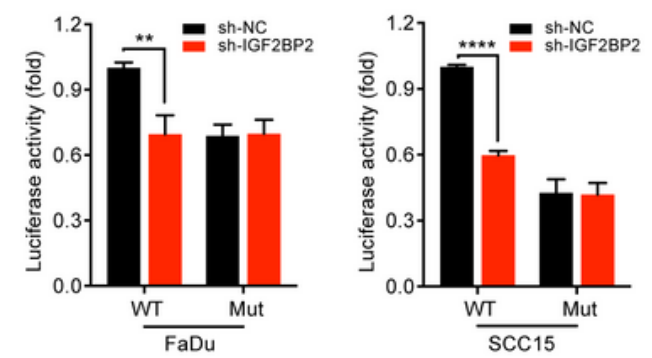

$\mathbf{F}$

FaDu
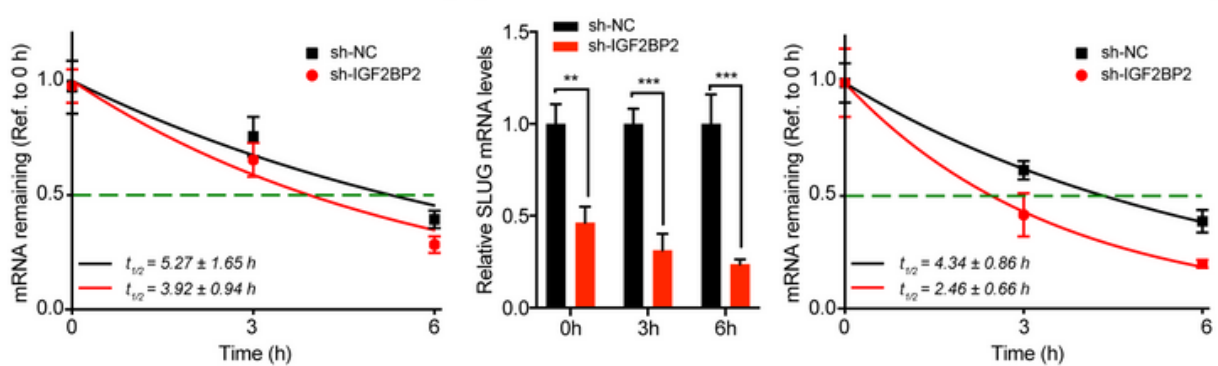

$\operatorname{scc} 15$

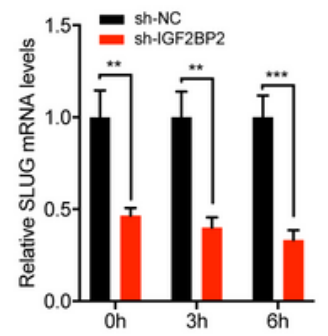

G

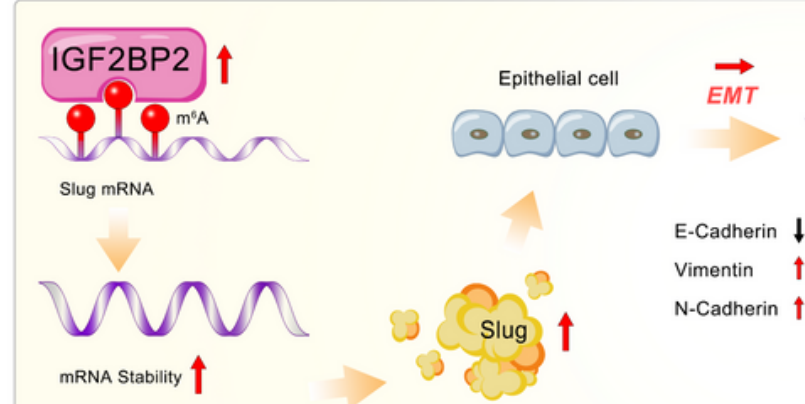

Cell motility and invasiveness $\uparrow$

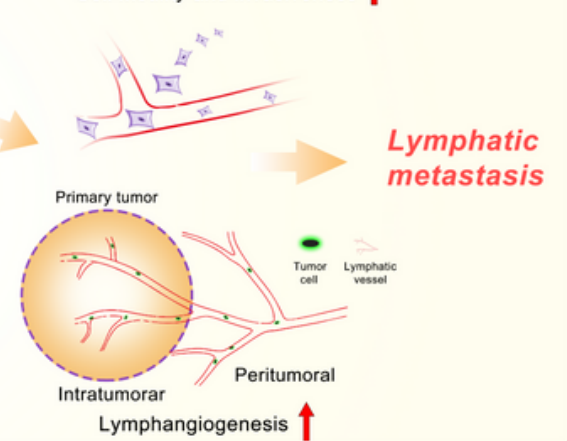

Figure 7

IGF2BP2 regulates slug mRNA stability via m6A modification (A) RIP-qPCR analysis showing the enrichment of Slug mRNA in anti-IGF2BP2 precipitates (left panel). Western blot detected the IGF2BP2 immunoprecipitation efficiency of IGF2BP2 RIP assay in FaDu and SCC15 cells (right panel). GAPDH and IgG served as an internal or negative control, respectively. ${ }^{* \star} P<0.001$. (B) MeRIP-qPCR analysis showing the m6A enrichment of Slug mRNA using anti-IgG and anti-m6A antibodies in FaDu and SCC15 cells after 
silencing IGF2BP2. ${ }^{*} P<0.01$. (C) A very high-confidence $m 6 A$ site was identified in the CDS region upon Slug mRNA based on the SRAMP software analysis. (D) Schematic representation of Slug- WT (wild-type) or Slug- MT (mutated-type) sequence. (E) Luciferase reporter assays measured the luciferase activities of Slug-CDS WT or Slug-CDS Mut in FaDu and SCC15 cells with IGF2BP2 knockdown. ${ }^{\star *} \mathrm{P}<0.01$, $\star \star \star \star P<0.0001$. (F) After silencing IGF2BP2 in FaDu and SCC15 cells, the mRNA half-lives and expression of Slug were analyzed at the predetermined times following actinomycin $D(5 \mu \mathrm{g} / \mathrm{ml})$ treatment. $(\mathrm{G}) \mathrm{A}$ graphic illustration of the proposed mechanism in this study. In brief, IGF2BP2 was highly expressed in HNSCC patients, and recognized and bound to the m6A site upon Slug mRNA to maintain its mRNA stability and expression, thereby promoting cell migration and invasion through EMT progress, and leading to the lymphatic metastasis in HNSCC. ${ }^{* *} \mathrm{P}<0.01$, ${ }^{\star \star *} \mathrm{P}<0.001$, ${ }^{\star \star \star *} \mathrm{P}<0.0001$.

\section{Supplementary Files}

This is a list of supplementary files associated with this preprint. Click to download.

- Additionalfile1.docx

- Additionalfile2.docx 